\title{
Non-Thermal Radio Frequency Stimulation of Tubulin Polymerization in Vitro: A Potential Therapy for Cancer Treatment
}

\author{
John T. Butters ${ }^{1}$, Xavier A. Figueroa ${ }^{2 *}$, Bennett Michael Butters ${ }^{1}$ \\ ${ }^{1}$ Nativis Inc., Seattle, WA, USA \\ ${ }^{2}$ Sciencia Incognita Consulting, LLC, Seattle, WA, USA \\ Email: ${ }^{\text {sci.figueroa@gmail.com }}$
}

Received 29 August 2014; revised 29 September 2014; accepted 28 October 2014

Copyright (C) 2014 by authors and Scientific Research Publishing Inc.

This work is licensed under the Creative Commons Attribution International License (CC BY). http://creativecommons.org/licenses/by/4.0/

c) (i) Open Access

\begin{abstract}
The use of radio frequency energy is an established technology for certain oncology therapies. Direct inputs of radio frequency (RF) energy as thermal energy are applied to ablate tumors or catalyze secondary reactions in adjunct treatments against certain tumor types. Yet, other applications are being developed which take advantage of properties of RFs that impinge on biological proteins and cells without thermal effects. Here we report a proof-of-concept application of specific, digitally encoded, low power (non-thermal) radio frequency energy in an in vitro preparation of a tubulin polymerization assay. The radio frequency energy signal, designated M2(3), was applied to the tubulin polymerization assay samples during spectrophotometric measurements to assess the effectiveness for enhancing tubulin polymerization. A commercially available taxane (paclitaxel) that promotes tubulin polymerization was used as a control to assess the effectiveness of the M2(3) radio frequency energy signal on tubulin polymerization rates. A low power, specific, digital radio frequency energy signal is capable of promoting tubulin polymerization as effectively as a commercially available taxane.
\end{abstract}

\section{Keywords}

Tubulin, Paclitaxel, Taxane, Radio Frequency Energy, Digitial Signal, Electromagnetic Energy, Microtubules, Non-Thermal, Polymerization, Cancer, Tumors

\section{Introduction}

The taxanes (e.g., paclitaxel, docetaxel) and vinka alkyloids (e.g., vinblastine, vinorelbine) are currently the only

\footnotetext{
*Corresponding author.
}

How to cite this paper: Butters, J.T., Figueroa, X.A. and Butters, B.M. (2014) Non-Thermal Radio Frequency Stimulation of Tubulin Polymerization in Vitro: A Potential Therapy for Cancer Treatment. Open Journal of Biophysics, 4, 147-168. http://dx.doi.org/10.4236/ojbiphy.2014.44015 
approved drugs for cancer chemotherapy that target mitosis by directly binding to the tubulin subunits [1]. Taxanes bind preferentially and reversibly to the $\beta_{1}$ subunit of tubulin in the microtubules. The binding of taxanes enhances polymerization of the tubulin into stable microtubules and inhibits microtubule depolymerization, inducing stable microtubule bundles, inhibiting cell division and inducing apoptosis [2]-[4]. The specificity of action of this drug has made it a useful and widely used chemotherapy agent in oncology.

Yet, the delivery of these drugs relies on the circulatory system to reach the target cells and tumor mass. In the case of solid tumors, drug penetration is incomplete due to poor blood vessel penetration, solubility of drug, organ metabolism and perfusion of the tumor [5] [6]. In the case of brain tumors, the blood-brain barrier (BBB) greatly inhibits the entry of a wide variety of drugs and molecules [7], making CNS localized cancers difficult to treat. The ability of cancer cells to exclude or develop resistance to the effects of the chemotherapy agents [8][12] reduces the efficacy of taxanes over time. More importantly, the systemic administration of these mitosis inhibitors targets non-cancerous cells and can produce a variety of side effects, which limit treatment options.

One potential therapy that has the characteristics of good tissue penetration, no induction of resistance to the drug therapy and low side effects is the use of radio frequency energy (RFE) therapy. RFE refers to the electromagnetic spectrum between $3 \mathrm{kHz}-3000 \mathrm{GHz}\left(\mathrm{ELF}-\mathrm{THF} / 10^{5} \mathrm{~km}-0.1 \mathrm{~mm}\right.$ ) that is derived from an alternating current (AC) source. Low power and low frequency RFE has good tissue penetration [13]-[15], ensuring access to areas that are poorly perfused. Ideally, therapies that have the characteristics of low power and low frequency RFE could produce better results for cancer treatments than drug-based approaches.

The rationale for using electrical or electromagnetic technologies in cancer therapy is not new. Radio frequency ablation of tumors [16]-[18] and targeted heating of tumor cells using RFE [19] are current applications. Low power electrical or EM technologies that rely on non-thermal effects are experimental treatment approaches for cancer therapies. For example a low-intensity alternating electrical field system [20]-[25] is undergoing a phase III clinical trial targeted against glioblastoma that relies on an electric field effect. The effects are nonthermal/non-chemical and appear to affect cells in telophase by "dielectrophoretic forces that act on polar and charged elements in the cell, pushing them toward the cytokinetic furrow leading to violent blebbing of the plasma membrane" [26].

A report by Berg et al. [27] reviews the published reports of low power (non-thermal), low frequency ( $25 \mathrm{~Hz}$ to $120 \mathrm{~Hz})$ and low magnetic intensity $(10-600 \mathrm{mT})$ EM fields in both cell culture and whole animal models. As reported, a pulsed/sinusoidal EMF coupled with or without chemotherapeutic agents produced an increase in apoptotic death for cancer cell lines in vitro and an inhibition in tumor growth in mouse models of cancer. Interestingly, no unifying mechanism was proposed to producing these repeatable effects.

Higher frequency systems $(55-75 \mathrm{GHz})$ with low power $\left(<1 \mathrm{~mW} / \mathrm{cm}^{2}\right)$ densities were able to induce an anti-proliferative effect in the 7932 RPMI human cancer cell line [28]. Ultra-structural and energy utilization analysis on human erythromyeloid leukemia cell line K562 in culture [29] revealed that cell membrane blebbing and an enhancement of the aerobic glycolytic pathway were probable causes for the decrease in cell proliferation. Although athermal in energy density, the mechanisms were thought to be due to vicinal cell-membrane water structural changes [29].

The investigational use of an intrabuccal EMF generating system has been described in increasing survival rates for terminal cancer patients [30], as well as helping to identify tumor types in affected individuals [31] [32]. Although preliminary and unorthodox, the results are repeatable and awaiting further confirmation in larger human trials. No mechanism was described.

Recently, Crocetti et al. [33] have demonstrated the ability to inhibit growth in the MCF7 breast cancer cell line using low power, low frequency pulsed EMF (20 - 50 Hz, 2 - $5 \mathrm{mT}$ ). This application selectively inhibited malignant cell lines (MCF7), but had no effect on the non-malignant cells (MCF10). Again, no mechanism was described.

The idea that low power, non-thermal RFE can be applied for cancer therapy is an expanding area for clinical research and treatment development. Here we report a proof-of-concept method on an acellular system for promoting the polymerization of tubulin subunits by using non-thermal radio frequency energy. Using a commercially available tubulin polymerization assay, we demonstrate that a non-thermal RFE signal can inhibit tubulin depolymerization and promote tubulin polymerization in much the same way as a taxane. The RFE signal, designated M2(3), was transmitted via paired Helmholtz coils to the tubulin samples during assays. Transmission of the signal was performed at 25 milliamps at a frequency of $22 \mathrm{kHz}$ (VLF, Band 2), with an average magnetic field strength of $0.1 \mathrm{nT}$ to $10 \mu \mathrm{T}$. A taxane was used as a positive control [34] to validate the results. Promotion 
of tubulin polymerization was observed under controlled conditions during M2(3) signal delivery and was confirmed via scanning electron microscopy. This RFE technology indicates the potential for use as a cancer therapy.

\section{Materials and Methods}

\subsection{Signal Acquisition and Selection}

A custom built electromagnetic isolation and recording chamber, called "Molecular Interrogation and Data System" (MIDS), was designed to measure time sequences of non-stimulated and DC offset magnetic field (MF) perturbations from molecules suspended in water (Supplement). Briefly, MIDS is a specialized, low temperature (liquid Helium) superconducting quantum interference device (SQUID), inside a shielded chamber, housing a low temperature shielded Dewar connected to a data acquisition system (Figure S1). The combination of the superconducting niobium second derivative gradiometer and the shielding in the MIDS is able to cancel external magnetic fields by $\sim 80 \mathrm{~dB}$ (dependent on frequency; Figure S3(a)). The low temperature SQUID coupled to the gradiometer detects small oscillations in the magnetic field over short time periods and converts these oscillations into current sequences. A DC offset $(226 \mathrm{mV})$ was injected to re-bias the SQUID when capturing signals from samples (ultra-pure [UP] water and the taxane in UP water). A taxane suspension (6 mg/mL; BMS Company, Princeton, NJ, NDC 0015-3475-30) in a glass vial (Kimble 60820-830, $8 \times 30 \mathrm{~mm}$ ) was lowered into the MIDS chamber. The magnetic field perturbations from a sample were recorded in the MIDS using a Keithley KPCI-3108 data acquisition board operating in differential mode. Signals were recorded for 60-second intervals with a $226 \mathrm{mV}$ offset, digitized using proprietary software (Supplement), and stored as a WAV file (Microsoft format) (Figure S3(b) and Figure S3(c) and Figure S4 for signals not selected).

The WAV file format is an industry standard for the recording and transmission of signals in the $0-40 \mathrm{kHz}$ range. Signals were selected from four sets of recordings done over a period of a few weeks (a total of 1059, 1minute recordings) from the MIDS, using a frequency analysis package in FlexPro8 (DEWETRON GmbH, Parkring 4, A-8074 Graz-Grambach, Germany). The digitized data from FlexPro8 plots of the signals were converted from the time domain into the frequency domain using a Fast Fourier Transform algorithm and then analyzed by plotting the spectrum after enhanced autocorrelation (see Supplement). The M2(3) signal was selected due to its high signal-to-noise, low interference and low entropy for testing in the tubulin polymerization assay.

\subsection{Signal Transmission and Coil Design}

Voyager, an in-house built amplifier, was used to deliver the digitized signal of (M2(3)) over an optical connection to an 8 Ohm Helmholtz coil embedded in a cuvette holder (see Figure S7(A) and Figure S7(B)). The optic cable from Voyager to the Helmholtz coil was used in order to deliver the highest fidelity signal with the lowest magnetic field fluctuation possible (Figure 1(c)). The M2(3) signal transmission was at levels of $0.1 \mu \mathrm{T}$ to 10 nT via a paired Helmholtz coil configuration (see Figure S7(A) and Figure S7(B)). The design of the coil was set to ensure uniformity of the field vector and to increase accuracy during playback of the signal. Modeling of the magnetic field was used in order to map the field strength and uniformity (see Figure S7(C)). The signal was transmitted as a complex wave form, with a variable flux density of 1-second duration at varying Tesla increments, using a program designed in LabView (National Instruments Corporation, Austin, TX).

\subsection{Mu-Metal Isolation Chamber}

All experiments were performed inside an EMF isolation chamber. Briefly, the chamber was constructed from 0.25-inch thick copper plate on all sides, surrounded via Mu-metal shielding on the inside and outside. The schematics covering this chamber are covered in Figure S6(a). A small hole on the side provided access for the cables and optics. All cables were twisted pair cables to minimize EMF interference. A small removable lid provided access to the Helmholtz coil and the cuvette holder (Figure S6(a) and Figure S6(b)).

\subsection{Tubulin Polymerization Assay}

All buffers, guanosine triphosphate (GTP), dimethyl sulfoxide (DMSO) and tubulin for the tubulin polymerization assay were purchased from Cytoskeleton, Inc. (http://www.cytoskeleton.com/; kit CDS01B). The lyophilized tubulin was resuspended in tubulin polymerization buffer (GPEM, which is PEM plus $1 \mathrm{mM}$ GTP and 5\% 
glycerol). PEM is the general tubulin buffer (80 mM Na-PIPES pH 6.9, $1 \mathrm{mM} \mathrm{MgCl}_{2}$ and $1 \mathrm{mM}$ EGTA). The tubulin solution was centrifuged at 13,000 rpm (Galaxy 14-D, VWR) for 10 minutes in $1.5 \mathrm{~mL}$ Eppendorf tubes and the supernatant was stored in aliquots of $160 \mu \mathrm{L}$ in $0.5 \mathrm{~mL}$ Eppendorf tubes in liquid nitrogen. The lyophilized tubulin was resuspended in tubulin polymerization buffer (GPEM) to a final concentration of $1.5 \mathrm{mg} / \mathrm{mL}$. All controls and experimental assays were performed at room temperature (RT) in a $70 \mu \mathrm{L}$ disposable, plastic cuvette (UV Cuvette, Plastibrand, GMBH + Co KG, Postach 11 55, 97861, Wethein, Germany) placed inside a $\mathrm{Mu}$-metal isolation chamber.

\subsection{Polymerization Experiments}

A spectrophotometer (Cary 50-Bio, Varian, North America) was used to measure optical density (OD) at 310 $\mathrm{nm}$ in kinetic mode, usually recorded as 10 min traces; absorbance was measured every 10 seconds and averaging time was set to 1 second. A fiber optic cable was used from the spectrophotometer to a custom built cuvette holder with Helmholtz coils (Figure 1(c), Figure 1(d); Figure S7(B)) placed inside the Mu-metal isolation chamber. Immediately before the start of an assay, an aliquot of the reaction mixture was thawed rapidly in an RT water bath. After a 10-minute spin (13,000 rpm; Galaxy 14-D, VWR) at $2^{\circ} \mathrm{C}$, the supernatant was transferred into a cuvette for continuous measurement at room temperature for $10 \mathrm{~min}$, unless otherwise indicated. Background polymerization (buffer control) was done in PEM buffer, polymerization reactions (taxane, tubulin control and M2(3) signal) were done in GPEM buffer. The samples were handled in sequence, starting with the buffer controls, followed by tubulin controls, then the taxane samples. The final samples in the sequence were exposed to the M2(3) signal. A single individual performed the assay runs for all the different samples and sample runs, in the same location and not deviating from the established sequence process. All samples experienced the same motion in space (handling, mixing and measurement) and temperatures were kept identical between samples. Temperature variations due to heating of the Helmholtz coils were sampled with signal power applied or without (Figure 2(e)). No discernible heating was observed when power was applied continuously to the coils over a 100 minute sampling run.

\subsection{Scanning Electron Microscopy Experiments}

All imaging was performed at NanoImaging Services, Inc., 10931 North Torrey Pines Road, Suite 108, San Diego, CA 92037. All samples were coded and were prepared for electron microscopy using negative staining by PTA (phosphotungstic acid, Ted Pella Inc.), $2 \%$ in $\mathrm{ddH}_{2} \mathrm{O}, \mathrm{pH}$ 7.12. Grids used were 400 copper mesh, coated with a layer of formvar and continuous carbon (Ted Pella Inc.). Grids were cleaned using a Solarus plasma cleaner (25\% O2, 75\% Ar; Gatan Warrendale, PA) for 10 seconds shortly before application of the sample. A 3 $\mu \mathrm{L}$ sample was applied to the surface of the grid. The sample was then washed using $5 \times 200 \mu \mathrm{L}$ drops of ddH2O sitting on the surface of parafilm. A $3 \mu \mathrm{L}$ drop of stain was then immediately added to the grid and allowed to sit for $\sim 30$ seconds. The grid was blotted by applying a KimWipe (Kimberly-Clark) to the edge of the grid and allowed to dry completely under ambient conditions. Electron microscopy was performed using an FEI Tecnai T12 electron microscope (FEI North America) operating at $120 \mathrm{KeV}$ equipped with an FEI Eagle $4 \mathrm{~K} \times 4$ K CCD camera (FEI North America). Images of each grid were acquired at multiple scales to assess the overall distribution of the specimen. After identifying potentially suitable target areas for imaging at lower magnifications, pairs of high magnification images were acquired at nominal magnifications of 52,000× $(0.21 \mathrm{~nm} / \mathrm{pixel})$ and $21,000 \times(0.50 \mathrm{~nm} \mathrm{~nm} /$ pixel). The images were acquired at a nominal under-focus of $-2 \mu \mathrm{m}(52,000 \times)$ and $-6 \mu \mathrm{m}(21,000 \times)$ and electron doses of $\sim 10-15 \mathrm{e}^{-} / \AA^{2}$.

\section{Results}

\subsection{Signal Acquisition and Transmission of the M2(3) Signal for Tubulin Assay}

Signals emitted by the taxane solution in the MIDS recording chamber were acquired over an 18 hour sampling run, with repeated one-minute measures, of the same sample with no DC offset and a range of DC offsets applied during recordings. A flow diagram (Figure 1) delineates the process of signal acquisition, signal processing and signal exposure to samples. Signals were analyzed from the recordings and sorted (Figure S2). 


\section{EM Signal of Molecule Recorded}

(a)

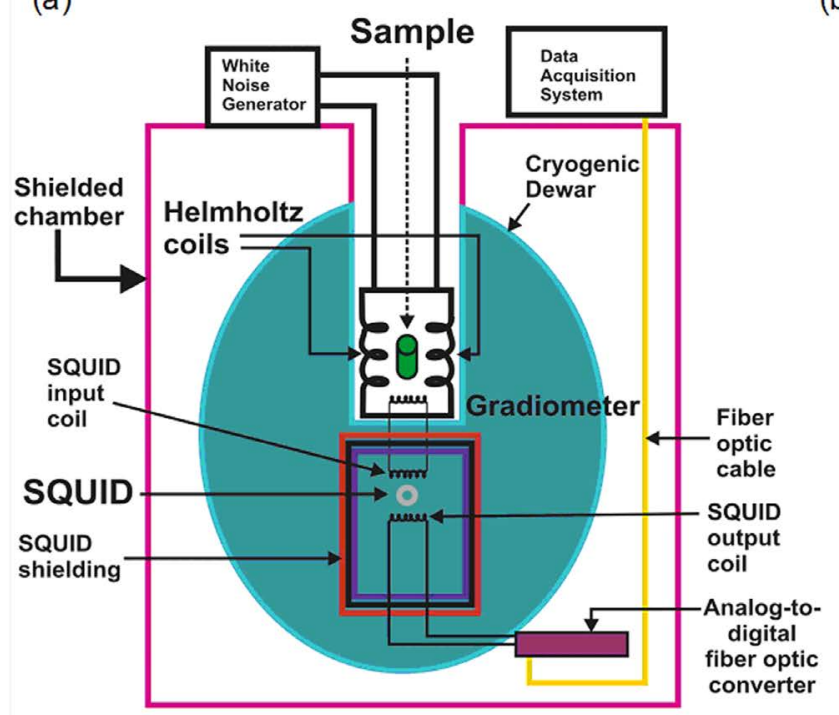

\section{Signal Analysis \& Digitization}

(b)
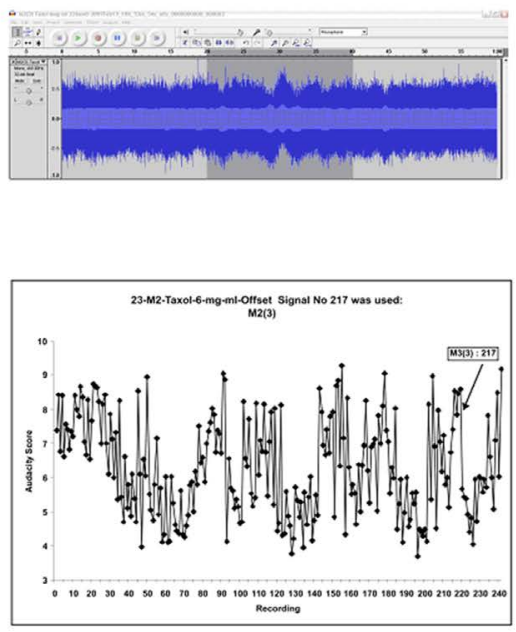

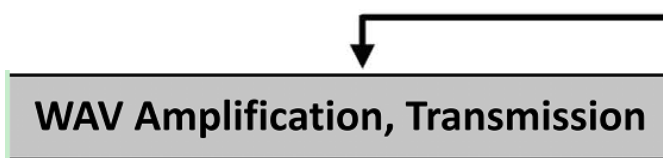

(c)

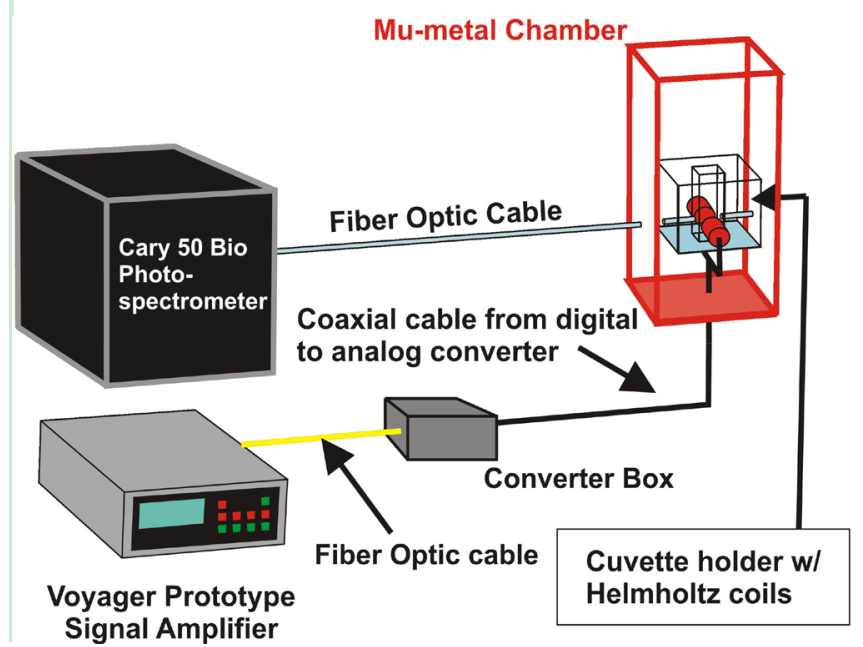

\section{Tubulin OD Assay Cuvette Holder}

(d)

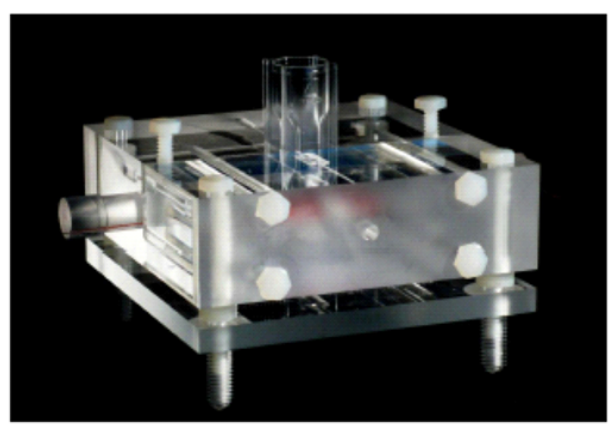

Figure 1. Flow schematic for signal acquisition, transmission and detection. (a) A magnetic field signal is acquired via cryogenic SQUID; (b) Analog signal (example) is analyzed and the radio frequency divided into signal components for digitization into a WAV file; (c) The WAV file is transmitted via an amplifier to an 8 Ohms Helmholtz coil embedded into a custom built cuvette holder; (d) The cuvette holder is specifically designed to accept a fiber optic cable for the measurement of optical density (OD) at $310 \mathrm{~nm}$ via a Cary-50 spectrophotometer. The cuvette holder is placed within a custom built Mumetal chamber, with only the fiber optic and amplifier wires running into the chamber.

The M2(3) signal was chosen as the likeliest candidate from the different samples. As explained in the Supplement, signals with high power-to-noise, low interference and low entropy were selected after analysis of the individual recordings from the MIDS. The digitized M2(3) signal was transduced with the Voyager to the Helm- 
holtz coil containing the cuvette with the tubulin solution in one-minute loops. These loops were emitted in 0.5 $\mathrm{dB}$ increments, 42 steps of one-second duration. All tubulin assays were performed inside the Mu-metal isolation chamber, ensuring that the only variable that changed was the induction of signal transmission over the Helmholtz pair producing a uniform gradient field.

\subsection{Tubulin Depolymerization Is Inhibited When Signal M2(3) Is Transmitted}

A total of 34 individual experiments were performed with the tubulin polymerization assay. All experimental runs were performed at room temperature $\left(23^{\circ} \mathrm{C}-25^{\circ} \mathrm{C}\right)$ in order to minimize spontaneous polymerization. Fresh aliquots from pre-made batches of the tubulin assay kit were thawed and used for each experiment. The cuvette holder (Figure 1(d)) was placed inside a custom built Faraday/Mu metal chamber (see Figure S6(a) and Figure S6(b)) with an access hatch for cuvette placement. During assays of taxane, tubulin control and buffer control, no signal was applied to the Helmholtz coils. The M2(3) signal was transmitted after the tubulin assay components were mixed in the cuvette, the cuvette placed in the holder and the lid closed.

Spectrophotometer OD traces measured over 10 minutes at $310 \mathrm{~nm}$ were recorded. All the traces from the 34 individual experiments are displayed in Figure 2, divided into 4 groups, A - D. All traces were set to zero by subtracting the starting value of a trace from all subsequent values along the trace. Traces were separated into 4 columns (buffer control, tubulin control, M2(3) signal and taxane) in order to better visualize the change in OD over time for each condition tested. In total, 111 replicate runs of buffer control, 72 replicate runs of tubulin control, 362 replicate runs of M2(3) signals (at varying magnetic intensities of $0.1 \mathrm{nT}$ to $10 \mu \mathrm{T}$ ) and 38 replicate runs of taxane $(4 \mu \mathrm{M})$ were done.

When the M2(3) signal was transmitted (Figures 2(a)-(d); M2(3) Signal column) during the tubulin polymerization assay, a discernible number of traces were captured with unique and repeatable response profiles, as compared to the tubulin control (Figures 2(a)-(d)) and buffer control (Figures 2(a)-(d)). Power to the Helmholtz coils was $\sim 25$ milliwatts, with temperature changes of $\pm 0.2^{\circ} \mathrm{C}$ during temperature sampling runs (Figure 2(e)) to the coils and cuvette. Changes in room temperature had greater effect on the set-up than did heating from the coils, but room temperature did vary approximately $\pm 0.7^{\circ} \mathrm{C}$.

Optical density traces for each experimental condition were separated into two groups (Figure 3(a)): 1) those that exceeded absolute OD changes of greater than 0.01 and 2) those that were less than OD 0.01. A subset of signals (M2(3) signal) produced a smooth profile of tubulin polymerization with sustained and rapid increase of the OD readings during the polymerization assay (Figure 3(a), thick green line). The M2(3) signal produced OD changes that were characteristic of tubulin depolymerization inhibition in 10.5\% (38/362) of the signals tested. In the taxane samples, inhibition of tubulin depolymerization occurred in 26.3\% (10/38) (Figure 3(a); Taxane column) of the samples. In the tubulin control sample, no spontaneous changes in OD readings were observed to exceed 0.01 units (Figure 3(a); Tubulin Control).

Although the majority of tubulin samples treated with the taxane or the M2(3) signal did not produce changes that were different from buffer and tubulin control samples (Figure 3(a), lower half), when the M2(3) signal did work, the traces were robust and easily identified from the background (Figure 3(a), upper half).

Trace averages were compared on the same graph (Figure 3(b)), clearly showing the M2(3) traces produced a higher response profile compared to the taxane traces. A one-way ANOVA $(\alpha=0.05)$ of the terminal OD measures (600 seconds) reveals a statistically significant difference between sample populations (Table 1). A posthoc T-Test (two-tailed, unequal variance; $\alpha=0.01$ ) with Bonferonni correction $(\mathrm{n}=6)$ revealed a statistical significance between the M2(3) OD > 0.01 and the other populations (Table 2). Although the tubulin polymerization assays were done at a sub-optimal temperature $\left(25^{\circ} \mathrm{C}\right.$ vs. $\left.37^{\circ} \mathrm{C}\right)$, the $\mathrm{M} 2(3)$ signal average was significantly higher than taxane (OD > 0.01) and had distinctly different velocity profiles (Figure 3(c)). Temperature variations were not sufficiently different (Figure 2(e)) to play a part in the measured differences among the M2(3) exposed samples, the taxane treated samples and the control samples.

The optical density profile of the M2(3) signal traces that exceeded changes of OD 0.01 produced (Figure $3(\mathrm{c})$ ) a higher and faster change in the OD readings than did taxane samples. The rate of change of the OD measurements over time appeared to decrease in accordance with the saturation of the samples with polymerizing tubulin and a depletion of freely available subunits. Both taxane treated samples and M2(3) signal exposed samples appeared to converge on a steady-state change in OD values after 10 minutes. The changes in OD values were further validated by electron microscopy. 
(a)
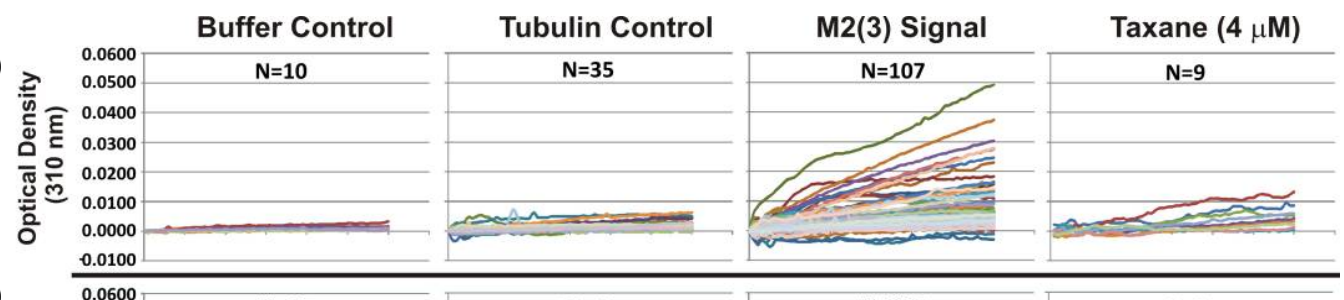

(b)
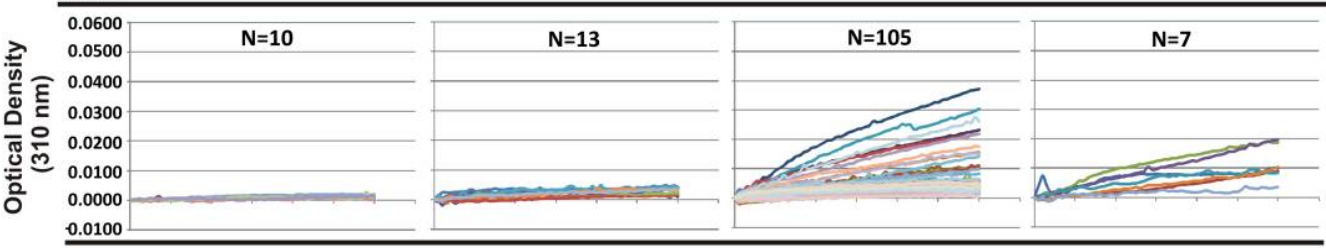

(c)
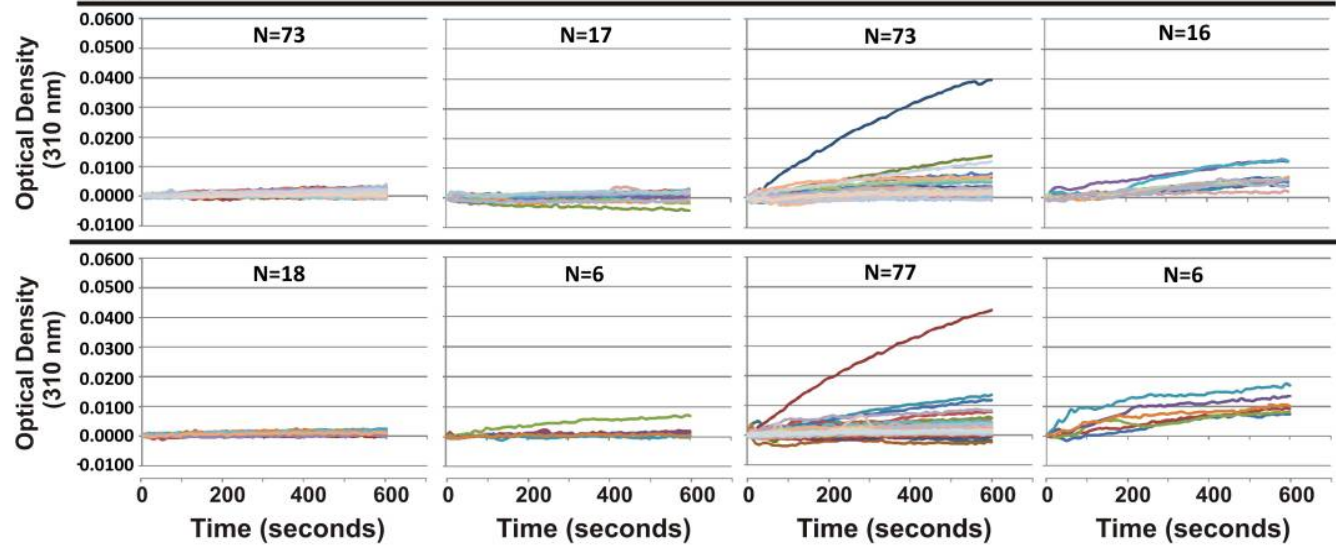

(d)

(e)

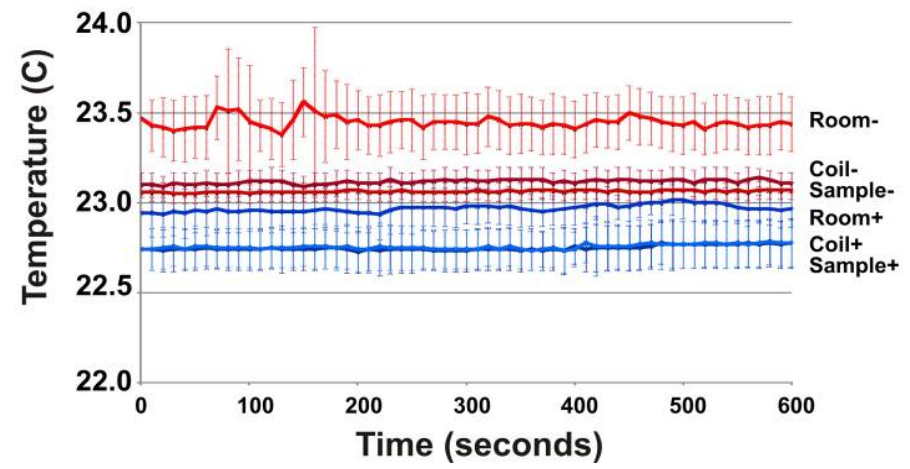

Figure 2. Tubulin polymerization assay traces. Graphs (a) 4 February 2008 to 14 August 2008; Graphs (b) 1 July 2008 to 3 February 2009; Graphs (c) 6 February 2009 to 25 February 2009; Graphs (d) 26 February 2009 to 6 March 2009. Experimental runs $(\mathrm{N}=34)$ are represented in the graphs; (e) Measurement of temperature variations during sample OD measurement with power on $(+)$ and power off $(-)$. Temperatures were measured with K-type resistors outside of the chamber (Room), on one of the Helmholtz coils (Coil) and inside the cuvette (Sample). Points are averages and error bars are standard deviation. ( $\mathrm{N}=10$ samples per condition).

\subsection{The M2(3) Signal Produces Stable Tubulin Structures under Scanning Electron Microscopy (SEM) Analysis}

Samples from a tubulin polymerization assay (Figure 4, SEM images) were taken after running the tubulin assay for 60 minutes. Samples were coded in order to blind the SEM technician as to the treatment of the samples. The micrographs are representative of the type of microtubules observed in selected visual fields of the SEM grid (Figures 4(A)-(F)). Tubulin exposed to the taxane $(4 \mu \mathrm{M})$ produced continuous microtubules of polymerized $\alpha$ and $\beta$ subunits of tubulin (Figure 4(A) and Figure 4(B)). In these controls, long, continuous bundles of microtubules were seen in a sparse background of small polymers or small aggregates of tubulin in the size range of $10-50 \mathrm{~nm}$. Very few microtubule bundles exceeded $100 \mathrm{~nm}$ in length. 


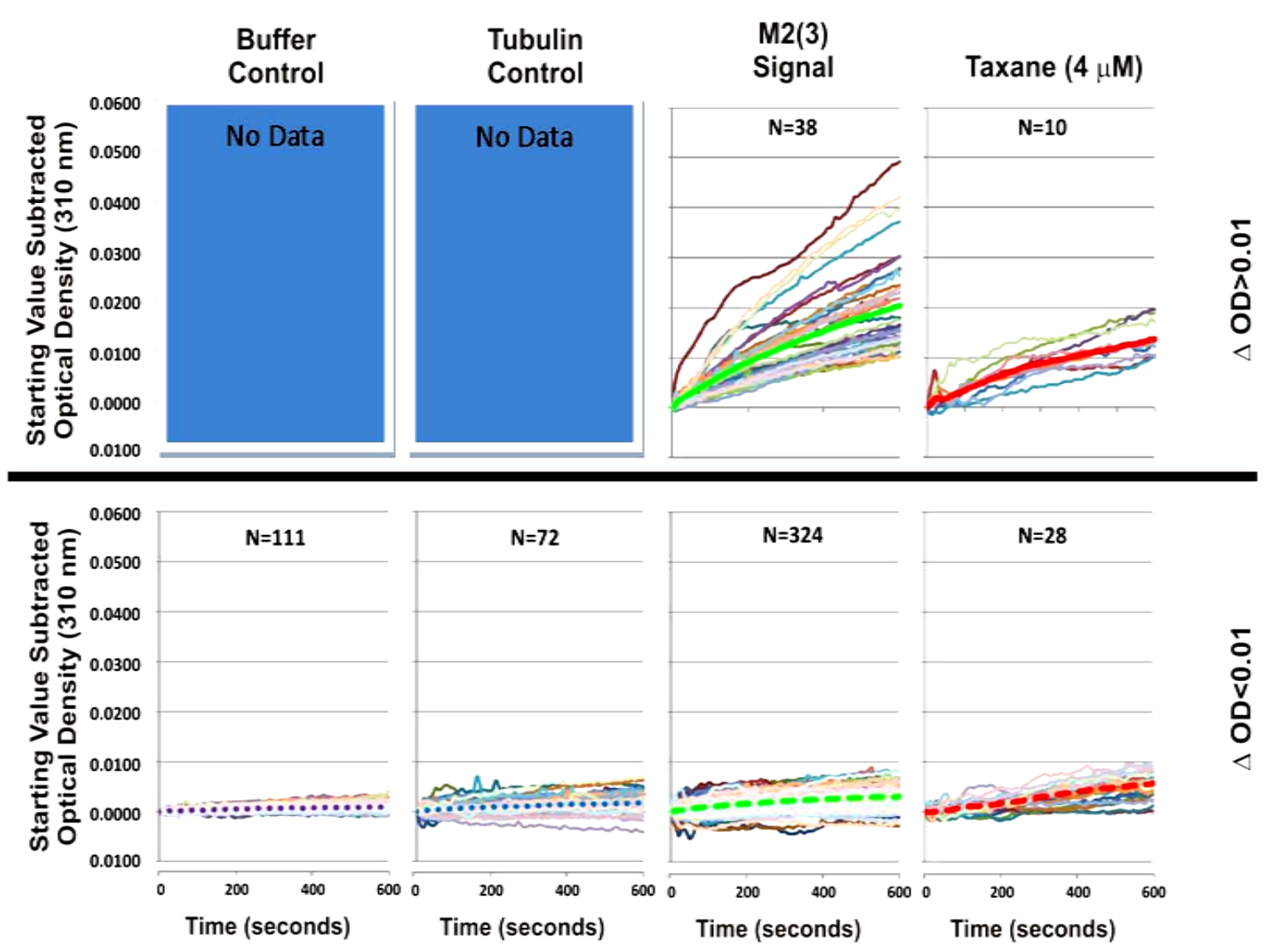

(a)

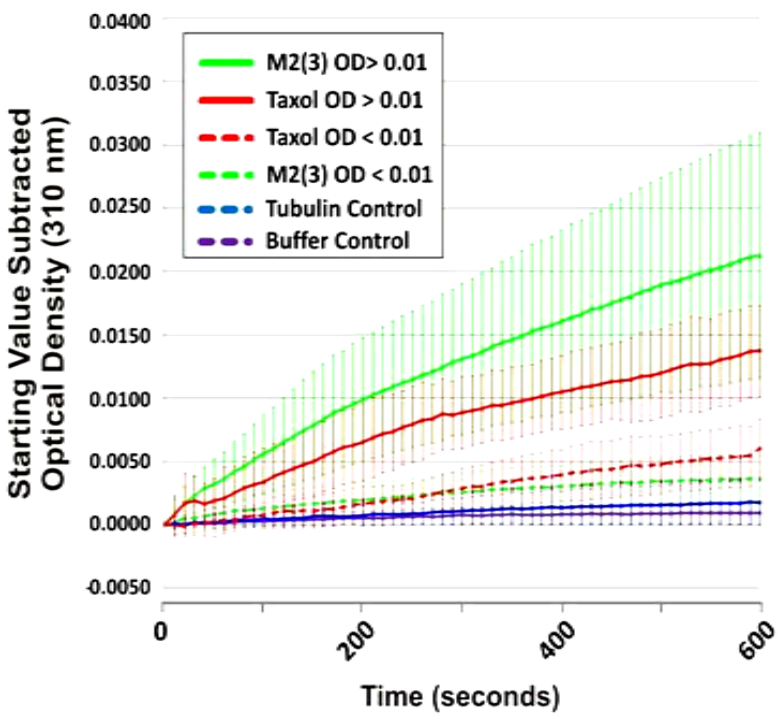

(b)

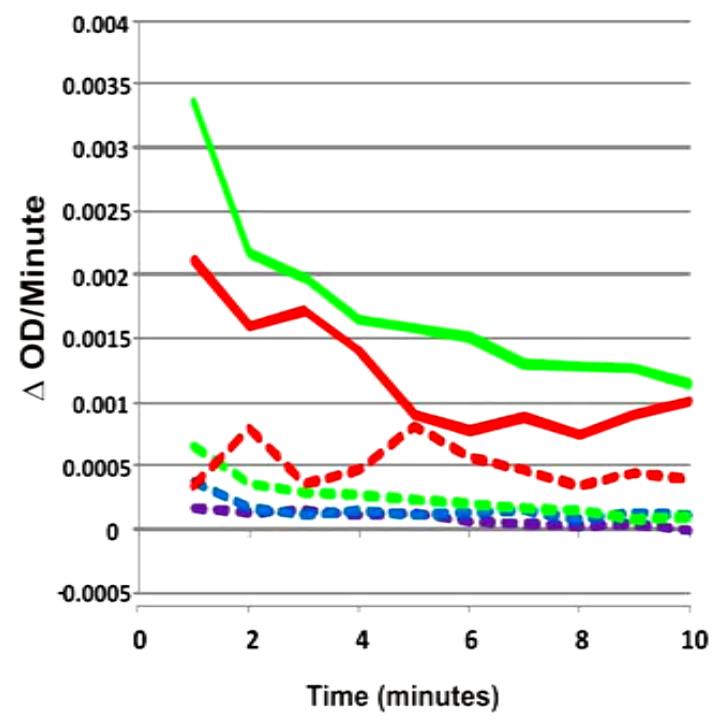

(c)

Figure 3. Tubulin polymerization graphs and velocity profiles. (a) Traces adjusted to a zero starting point are plotted on optical density (OD) versus time (seconds) plots. The two groups (upper and lower graphs) represent traces that are separated by total change in optical density measures $(\Delta>0.01$, upper graphs; $\Delta<0.01$, lower graphs). Thick lines are the averages of graphs $\Delta>0.01$. Dashed lines are the average of the total traces on each graph of $\Delta<0.01$; (b) Average values from each treatment group, separated into traces that exceeded OD changes (solid lines) of 0.01 or traces that did not exceed OD changes (dashed lines) of 0.01 . Error bars are standard deviation; (c) Plot of the average velocity profiles $(\Delta \mathrm{OD} / \mathrm{minute})$ for each group in graph b. Color and line styles represent the same groups as in graph $\mathrm{b}$. 
Table 1. Results of a single factor analysis of variance for the optical density measures from the tubulin polymerization experiments.

\begin{tabular}{cccccccc}
\hline \multicolumn{7}{c}{ ANOVA: Single Factor } \\
\hline Groups & Count & Sum & Average & Variance & SD & SD Low & SD High \\
\hline Buffer & 111 & 0.11 & 0.0010 & $8.51 \mathrm{E}-07$ & 0.0009 & $3.7 \mathrm{E}-05$ & 0.002 \\
Tubulin Control & 72 & 0.14 & 0.0018 & $4.31 \mathrm{E}-06$ & 0.0021 & $-3.0 \mathrm{E}-04$ & 0.004 \\
M2(3) OD $<0.01$ & 324 & 0.90 & 0.0030 & $2.74 \mathrm{E}-06$ & 0.0018 & $1.1 \mathrm{E}-03$ & 0.005 \\
M2(3) OD $>0.01$ & 38 & 0.77 & 0.0203 & $9.33 \mathrm{E}-05$ & 0.0098 & $1.1 \mathrm{E}-02$ & 0.030 \\
Taxol OD $<0.01$ & 28 & 0.17 & 0.0060 & $5.38 \mathrm{E}-06$ & 0.0023 & $3.7 \mathrm{E}-03$ & 0.008 \\
Taxol OD $>0.01$ & 10 & 0.14 & 0.0137 & $1.27 \mathrm{E}-05$ & 0.0036 & $1.0 \mathrm{E}-02$ & 0.017 \\
ANOVA: Single Factor & $\Delta=0.05$ & & & & & F crit & \\
Source of Variation & SS & $d f$ & MS & & & \\
Between Groups & 0.013 & 5 & 0.002584 & 291.5 & $4.0 \mathrm{E}-153$ & & 2.23 \\
Within Groups & 0.005 & 578 & 0.000009 & & & & \\
Total & 0.018 & 583 & & & &
\end{tabular}

Table 2. Results of a two-tailed t-Test comparing the M2(3) OD > 0.01 measures to all the other measures in our experiments.

\begin{tabular}{ccccc}
\hline & t-Test: Two-Sample Assuming Unequal Variances & & \\
\hline Alpha $=0.01$ & Samples & Mean & $\mathrm{P}(\mathrm{T}<=$ t) two-tail \\
\hline Bonferonni $=0.001667$ & M2(3) OD $>0.01$ & 0.0203 & & \\
Significant $(*)$ & Taxol OD $>0.01$ & 0.0137 & $1.4 \mathrm{E}-03$ & $*$ \\
& Taxol OD $<0.01$ & 0.0060 & $3.8 \mathrm{E}-11$ & $*$ \\
& M2(3) OD $<0.01$ & 0.0030 & $2.8 \mathrm{E}-13$ & $*$ \\
Tubulin Control & 0.0018 & $2.4 \mathrm{E}-14$ & $1.1 \mathrm{E}-14$ & $*$ \\
\hline
\end{tabular}

When samples subjected to the M2(3) signal were analyzed, the high concentrations of these protein aggregates obscured many of the features of interest. Several large polymeric structures were seen (some in the order of $1 \mu \mathrm{m}$ in length), but details were obscured by the high background in many areas of the grid. Structures that could be seen (Figure 4(C) and Figure 4(D)) reveal highly aggregated bundles of what are apparently microtubules. Large polymeric structures were seen more frequently with M2(3) signal exposed samples than with the taxane treated samples. Both the tubulin control and white noise control reveal a sparse background of small polymers and aggregates, with no large polymeric structures appearing in any of the fields that were visualized. The SEM imaging results were consistent with the observed tubulin assays.

\section{Discussion}

The electromagnetic spectrum encompasses a wide range of phenomena, from gamma rays to radio waves. Our increasing understanding of the effects of the EM spectrum in biology will likely render new options for cancer treatment in the near future. The development of RFE for therapeutic applications is still at an early stage, with thermal ablation being an early application. Other technologies, like the NovoCure ${ }^{\mathrm{TM}}$ tumor treating field (TTF) using alternating electric fields to treat cerebral tumors [22], are early indicators of the direction for RFE- and EM-based therapies in oncology.

The understanding of EMF's direct interaction with biological processes has undergone a transition in the last thirty years. The weight of evidence clearly supports the hypothesis that EMF and RFE can directly interact and affect biological proteins and alter biological processes without thermal effect [35]-[38]. The question of how the EMF or RFE interacts with the biological machinery still remains to be understood and answered. Depending 

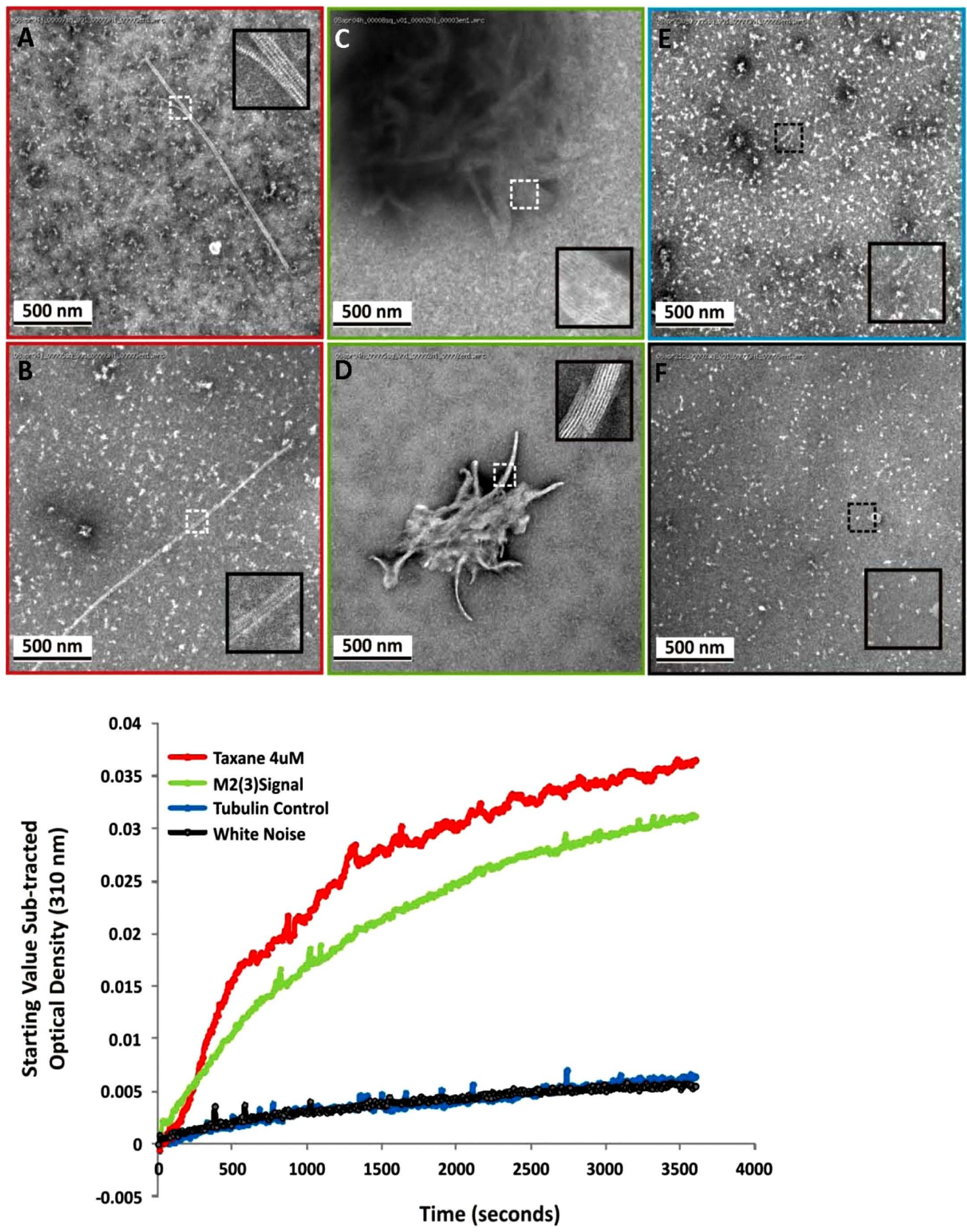

Figure 4. Scanning electron microscopy (SEM) of tubulin polymerization assay. Samples taken from an experiment using taxane (A and B), M2(3) signal (C and D), GTP control (E) and a white noise control (F) runs. Micrographs are all magnified $21,000 \times$, while insets are enlarged and zoomed micrographs taken at 52,000×. Dashed boxes represent the area of interest for the inset. The graph to the right of the micrographs is the tubulin polymerization assays from which the samples were taken for SEM imaging. Samples were taken at the end of the assay runs. Colored outlines around the micrograph represent the lines of the graph from which the samples were derived. 
on the frequency used, different effects of low power RF energy produce either growth inhibition of tumor cell lines or increased survival rates in tumor-bearing mice [27] [28] [39]-[43].

The underlying theory for the Nativis RFE technology is the ability to mimic the magnetic environment that surrounds a solvated taxane molecule (or group of molecules). By measuring the magnetic signal of taxane molecules in a water suspension, digitizing the output and transmitting an RFE signal to our samples, we hypothesized that we could mimic specific magnetic components of the taxane and redistribute charge via the water molecules around the solvated $\alpha-\beta$ tubulin proteins. We hypothesize that the RFE signal (M2(3) signal) stabilized the tubulin subunits by redistributing charge via the magnetic field of the RFE in solvated proteins [44], similar to the charge distribution that a physical taxane molecule induces. Electron transfer is central to the function of many biologic processes and artificial magnetic fields are capable of triggering a receptor response and conformational change in the absence of a physical agonist [45]. We suspect that the RFE interacts with the solvation shell surrounding the tubulin subunit, causing the solvation shell to increase in density, forcing a conformational change [46]. The energy range used in these experiments is in the milliwatt range, producing magnetic fields of $0.1 \mathrm{nT}$ to $10 \mu \mathrm{T}$ intensities. These energy levels are well below thermal ablation intensities, but they are within the range capable of affecting biological proteins [47].

Our rationale for using the tubulin polymerization assay as a proof-of-concept was the well characterized nature of the assay, its high repeatability, its use in industry to test the effects of new molecules on potential cellcycle inhibition, a completely acellular system and the minimum number of protein components [48]-[50]. The M2(3) signal was delivered via a digitally controlled prototype amplifier unit (Voyager, Figure S6(b)) and there was no variation in the M2(3) signal content. Indeed, these experiments represent the earliest attempts by Nativis to develop the proof-of-concept technology for RFE transmission of specific signals, using prototype systems that were not optimized.

Although temperature plays a critical role in this assay, we consciously chose to perform the tubulin polymerization assay at RT. According to the tubulin assay manufacturer's instruction (Cytoskeleton, Cat. \# BK006P), every $1{ }^{\circ} \mathrm{C}$ drop in temperature from $37^{\circ} \mathrm{C}$ produces a $5 \%$ drop in OD change. A 50\% drop in OD change potential from the manufacturer's estimates was calculated to have occurred in our experimental setup, reducing spontaneous polymerization of tubulin. We suspect that the low number of taxane traces that exceeded OD > 0.01 were due to this low temperature regime. The low temperature regime makes the observation of increased polymerization with the M2(3) signals more notable. Concerns regarding thermal effects of the RFE are minor as the energy range used was not sufficient to raise the temperature of the coils or alter the temperature close to the samples. Measured temperature changes with power applied to the coils resulted in less than $\pm 0.2^{\circ} \mathrm{C}$ change over 2 - 3 hours (Figure 2(e)). Both the coil and sample temperatures varied minimally and do not explain the observed difference in OD.

All traces were displayed in order to show the variability within the experimental system, as well as the effect of the M2(3) signal on tubulin polymerization. All traces were screened under strict criteria and analyzed independently of the scientists at Nativis, independently by an external reviewer (XAF). The variation of the traces for the M2(3) signal was probably due to non-optimized conditions, as a range of Tesla intensities were applied during exposure to tubulin samples $(0.1 \mathrm{nT}$ to $10 \mu \mathrm{T}$ ). Experimental sets were selected and analyzed only when control samples (buffer and tubulin controls) were at steady state conditions (no changes in OD over 10 minutes). Otherwise the entire experimental set (controls, M2(3) signal and taxane) was excluded in the analysis. Data in aggregate can become meaningless when discussing a new technology that is non-optimized, hence our decision to divide signals into two groups ( $\mathrm{OD}>0.01$ and $\mathrm{OD}<0.01$ ). The hit-rate (i.e., the tubulin assay exceeding $\mathrm{OD}>$ 0.01 ) was $10.5 \%$ for the M2(3) signal, less than half of the taxane group (26.3\%), but greater than the tubulin control group (no events exceeding OD $>0.01$ ). Furthermore, the overall OD changes were statistically significant for the M2(3) signal that exceeded OD $>0.01$.

The characteristic of the OD changes when the M2(3) signals were delivered to the tubulin samples was the rapid rise in OD measurements, with a stable nucleation and growth phase (Figure 3(b)) that was greater than the taxane positive control. The taxane treated samples showed a characteristically less stable nucleation phase (there was a drop in OD values within 25 - 50 seconds of the start of measurement) and a slower and lower value transition into the growth phase (Figure 3(b)). Measurements of the rate in OD change also demonstrated that the samples exposed to the M2(3) signal produced larger and more sustained rates of change than the taxane treated samples (Figure 3(c)).

Further supporting evidence is provided by the use of scanning electron microscopy on samples that were 
prepared after an experimental run with the M2(3) signal. Control samples, as well as white noise (random signal RFE transmission), revealed globular and sparse deposits on the SEM images (Figure 4(E), Figure 4(F)). Only the M2(3) signal samples (Figure 4(C), Figure 4(D)) and taxane sample (Figure 4(A), Figure 4(B)) revealed any polymeric structures that could be recognized as microtubules. Furthermore, the M2(3) signal produced an excess of short, globular polymers and highly aggregated microtubule bundles. These were not seen in any of the other samples, including the white noise samples. The addition of white noise to this set was to preclude a thermal effect for the SEM portion of our experiments. White noise was not included as a control of the previous sets (Figure 2 and Figure 3) of OD measures, mainly due to time constraints and the lack of change in temperature that was observed (Figure 2(e)) when the M2(3) signal was transmitted. The SEM samples were also analyzed independently by a third-party specialist (NanoImaging Services). The samples were coded so that the SEM technician would not know the type of treatment the sample received and the subsequent interpretation would not be contaminated by the expectations of the Nativis scientists.

Our study strongly supports the hypothesis that a specifically encoded, digitally controlled, non-thermal RFE signal can induce changes in tubulin subunit interactions that mimic those of a taxane. A key concept to keep in mind is the specificity of the signal that produces the effect and the cyclical exposure of the signal to the samples being analyzed. Furthermore, this is the first published use of an RF technology that directly measures the effect on a cell-free system and targets specific proteins ( $\alpha-\beta$ tubulin dimers). Non-specific effects due to cellmembrane impedance or $\mathrm{Ca}^{2+}$ released from intracellular organelles cannot explain the inhibition of tubulin depolymerization.

Although arguments can be made that our testing, at best, biased the promotion of tubulin polymerization, we contend that the RFE signal can be developed into a medically relevant technology. Based on these proof-ofconcept results, we are embarking on cell culture and mouse studies of U-87 GM cells lines. We will test the effectiveness and efficacy with a more advanced version of the Voyager system and with an independent laboratory performing the experiments and analysis.

\section{Acknowledgements}

We would like to thank the staff and scientist at Tristan Technologies, San Diego, CA, Douglas Paulson, Ph.D., Michael Leonard, M.S., J.D. and Robert Fagaly, Ph.D. and many other staff members that helped in this project. We would like to thank Dr. Gabriel Vogeli and Mr. Steven Pope for their review and comments on the manuscript. We declare that the authors of this manuscript have a conflict of interest with this publication. John T. Butters and Bennett Michael Butters are the CEO and Vice President-Signal Technology, respectively, of Nativis, Inc., a privately held company incorporated in the state of Washington, U.S.A. Both are shareholders of Nativis, Inc. where the current work has been performed. Dr. Xavier A. Figueroa declares a conflict of interest. He is a contracted consultant of Nativis, Inc. via Sciencia Incognita Consulting, LLC (Washington, U.S.A.). Dr. Figueroa has reviewed the data, analyzed the results and prepared the manuscript.

\section{References}

[1] Jordan, M.A. and Wilson, L. (2004) Microtubules as a Target for Anticancer Drugs. Nature Reviews Cancer, 4, 253265. http://dx.doi.org/10.1038/nrc1317

[2] Agrawal, N.R., Ganapathi, R. and Mekhail, T. (2003) Tubulin Interacting Agents: Novel Taxanes and Epothilones. Current Oncology Reports, 5, 89-98. http://dx.doi.org/10.1007/s11912-003-0095-6

[3] Wang, T.H., Wang, H.S. and Soong, Y.K. (2000) Paclitaxel-Induced Cell Death: Where the Cell Cycle and Apoptosis Come Together. Cancer, 88, 2619-2628. http://dx.doi.org/10.1002/1097-0142(20000601)88:11<2619::AID-CNCR26>3.0.CO;2-J

[4] Verweij, J., Clavel, M. and Chevalier, B. (1994) Paclitaxel (Taxol) and docetaxel (Taxotere): Not Simply Two of a Kind. Annals of Oncology, 5, 495-505.

[5] Lammers, T., et al. (2012) Drug Targeting to Tumors: Principles, Pitfalls and (Pre-) Clinical Progress. Journal of Controlled Release, 161, 175-187. http://dx.doi.org/10.1016/j.jconrel.2011.09.063

[6] Corti, A., et al. (2012) Targeted Drug Delivery and Penetration into Solid Tumors. Medicinal Research Reviews, 32, 1078-1091. http://dx.doi.org/10.1002/med.20238

[7] Deeken, J.F. and Loscher, W. (2007) The Blood-Brain Barrier and Cancer: Transporters, Treatment, and Trojan Horses. Clinical Cancer Research, 13, 1663-1674. http://dx.doi.org/10.1158/1078-0432.CCR-06-2854 
[8] Orr, G.A., et al. (2003) Mechanisms of Taxol Resistance Related to Microtubules. Oncogene, 22, 7280-7295. http://dx.doi.org/10.1038/sj.onc.1206934

[9] Yusuf, R.Z., et al. (2003) Paclitaxel Resistance: Molecular Mechanisms and Pharmacologic Manipulation. Current Cancer Drug Targets, 3, 1-19. http://dx.doi.org/10.2174/1568009033333754

[10] Kavallaris, M., Annereau, J.P. and Barret, J.M. (2008) Potential Mechanisms of Resistance to Microtubule Inhibitors. Seminars in Oncology, 35, S22-S27. http://dx.doi.org/10.1053/j.seminoncol.2008.01.006

[11] Kinsella, A.R., Smith, D. and Pickard, M. (1997) Resistance to Chemotherapeutic Antimetabolites: A Function of Salvage Pathway Involvement and Cellular Response to DNA Damage. British Journal of Cancer, 75, 935-945. http://dx.doi.org/10.1038/bjc.1997.164

[12] Zhou, X.J. and Rahmani, R. (1992) Preclinical and Clinical Pharmacology of Vinca Alkaloids. Drugs, 44, 1-16. http://dx.doi.org/10.2165/00003495-199200444-00002

[13] Robitaille, P.M., Kangarlu, A. and Abduljalil, A.M. (1999) RF Penetration in Ultra High Field MRI: Challenges in Visualizing Details within the Center of the Human Brain. Journal of Computer Assisted Tomography, 23, 845-849. http://dx.doi.org/10.1097/00004728-199911000-00006

[14] Roschmann, P. (1987) Radiofrequency Penetration and Absorption in the Human Body: Limitations to High-Field Whole-Body Nuclear Magnetic Resonance Imaging. Medical Physics, 14, 922-931. http://dx.doi.org/10.1118/1.595995

[15] Bottomley, P.A. and Andrew, E.R. (1978) RF Magnetic Field Penetration, Phase Shift and Power Dissipation in Biological Tissue: Implications for NMR Imaging. Physics in Medicine and Biology, 23, 630-643.

[16] Conzo, G., Sciascia, V., Palazzo, A., Stanzione, F., Pietra, C.D., Insabato, L., et al. (2013) Radiofrequency-Assisted Partial Nephrectomy for Metanephric Adenoma: A Case Report and Literature Review. Surgical Innovation, 20, 55-58. http://dx.doi.org/10.1177/1553350612442792

[17] Schutt, D.J. and Haemmerich, D. (2008) Tumor Ablation at Low Frequencies for Preferential Tumor Heating: Initial Ex-Vivo Tissue Studies. Proceedings of 30th Annual International Conference of the IEEE Engineering in Medicine and Biology Society, EMBS 2008, Vancouver, 20-25 August 2008, 238-241.

[18] Belfiore, G., Tedeschi, E., Ronza, F.M., Belfiore, M.P., Volpe, T.D., Zeppetella, G., et al. (2008) Radiofrequency Ablation of Bone Metastases Induces Long-Lasting Palliation in Patients with Untreatable Cancer. Singapore Medical Journal, 49, 565-570.

[19] Schmidt, C. (2008) The Kanzius Machine: A New Cancer Treatment Idea from an Unexpected Source. Journal of the National Cancer Institute, 100, 985-986. http://dx.doi.org/10.1093/jnci/djn246

[20] Chen, H., Liu, R., Liu, J. and Tang, J. (2012) Growth Inhibition of Malignant Melanoma by Intermediate Frequency Alternating Electric Fields, and the Underlying Mechanisms. Journal of International Medical Research, 40, 85-94. http://dx.doi.org/10.1177/147323001204000109

[21] Mrugala, M.M., Adair, J.E. and Kiem, H.P. (2012) Outside the Box-Novel Therapeutic Strategies for Glioblastoma. Cancer Journal, 18, 51-58. http://dx.doi.org/10.1097/PPO.0b013e318243f785

[22] Pless, M. and Weinberg, U. (2011) Tumor Treating Fields: Concept, Evidence and Future. Expert Opinion on Investigational Drugs, 20, 1099-1106. http://dx.doi.org/10.1517/13543784.2011.583236

[23] Kirson, E.D., Giladi, M., Gurvich, Z., Itzhaki, A., Mordechovich, D., Schneiderman, R.S., et al. (2009) Alternating Electric Fields (TTFields) Inhibit Metastatic Spread of Solid Tumors to the Lungs. Clinical \& Experimental Metastasis, 26, 633-640. http://dx.doi.org/10.1007/s10585-009-9262-y

[24] Schroeder, T., Viglianti, B.L. and Dewhirst, M.W. (2008) Low-Intensity Alternating Electric Fields: A Potentially Safe and Effective Treatment of Cancer? Onkologie, 31, 357-358. http://dx.doi.org/10.1159/000140601

[25] Kirson, E.D., Dbalý, V., Tovaryš, F., Vymazal, J., Soustiel, J.F., Itzhaki, A., et al. (2007) Alternating Electric Fields Arrest Cell Proliferation in Animal Tumor Models and Human Brain Tumors. Proceedings of the National Academy of Sciences of the United States of America, 104, 10152-10157. http://dx.doi.org/10.1073/pnas.0702916104

[26] Gutin, P.H. and Wong, E.T. (2012) Noninvasive Application of Alternating Electric Fields in Glioblastoma: A Fourth Cancer Treatment Modality. American Society of Clinical Oncology Educational Book, 32, 126-131.

[27] Berg, H., Günther, B., Hilger, I., Radeva, M., Traitcheva, N. and Wollweber, L. (2010) Bioelectromagnetic Field Effects on Cancer Cells and Mice Tumors. Electromagnetic Biology and Medicine, 29, 132-143. http://dx.doi.org/10.3109/15368371003776725

[28] Beneduci, A., Chidichimo, G., De Rose, R., Filippelli, L., Straface, S.V. and Venuta, S. (2005) Frequency and Irradiation Time-Dependant Antiproliferative Effect of Low-Power Millimeter Waves on RPMI 7932 Human Melanoma Cell Line. Anticancer Research, 25, 1023-1028.

[29] Beneduci, A., Chidichimo, G., Tripepi, S., Perrotta, E. and Cufone, F. (2007) Antiproliferative Effect of Millimeter Radiation on Human Erythromyeloid Leukemia Cell line K562 in Culture: Ultrastructural- and Metabolic-Induced Changes. Bioelectrochemistry, 70, 214-220. http://dx.doi.org/10.1016/j.bioelechem.2006.07.008 
[30] Barbault, A., Costa, F.P., Bottger, B., Munden, R.F., Bomholt, F., Kuster, N., et al. (2009) Amplitude-Modulated Electromagnetic Fields for the Treatment of Cancer: Discovery of Tumor-Specific Frequencies and Assessment of a Novel Therapeutic Approach. Journal of Experimental \& Clinical Cancer Research, 28, 51. http://dx.doi.org/10.1186/1756-9966-28-51

[31] Zimmerman, J.W., Jimenez, H., Pennison, M.J., Brezovich, I., Morgan, D., Mudry, A., et al. (201) Targeted Treatment of Cancer with Radiofrequency Electromagnetic Fields Amplitude-Modulated at Tumor-Specific Frequencies. Chinese Journal of Cancer, 32, 573-581.

[32] Zimmerman, J.W., Pennison, M.J., Brezovich, I., Yi, N., Yang, C.T., Ramaker, R., et al. (2012) Cancer Cell Proliferation Is Inhibited by Specific Modulation Frequencies. British Journal of Cancer, 106, 307-313. http://dx.doi.org/10.1038/bjc.2011.523

[33] Crocetti, S., Beyer, C., Schade, G., Egli, M., Fröhlich, J. and Franco-Obregón, A. (2013) Low Intensity and Frequency Pulsed Electromagnetic Fields Selectively Impair Breast Cancer Cell Viability. PLoS ONE, 8, e72944. http://dx.doi.org/10.1371/journal.pone.0072944

[34] Mitra, A. and Sept, D. (2008) Taxol Allosterically Alters the Dynamics of the Tubulin Dimer and Increases the Flexibility of Microtubules. Biophysical Journal, 95, 3252-3258. http://dx.doi.org/10.1529/biophysj.108.133884

[35] Goodman, R., Lin-Ye, A., Geddis, M.S., Wickramaratne, P.J., Hodge, S.E., Pantazatos, S.P., et al. (2009) Extremely Low Frequency Electromagnetic Fields Activate the ERK Cascade, Increase hsp70 Protein Levels and Promote Regeneration in Planaria. International Journal of Radiation Biology, 85, 851-859.

[36] Blank, M. and Goodman, R. (2009) Electromagnetic Fields Stress Living Cells. Pathophysiology, 16, 71-78. http://dx.doi.org/10.1016/j.pathophys.2009.01.006

[37] Blank, M. (2005) Do Electromagnetic Fields Interact with Electrons in the Na,K-ATPase? Bioelectromagnetics, 26, 677-683. http://dx.doi.org/10.1002/bem.20167

[38] Blank, M. and Goodman, R. (2004) Initial Interactions in Electromagnetic Field-Induced Biosynthesis. Journal of Cellular Physiology, 199, 359-363. http://dx.doi.org/10.1002/jcp.20004

[39] Ř́íhová, B., Etrych, T., Šírová, M., Tomala, J., Ulbrich, K. and Kovář, M. (2011) Synergistic Effect of EMF-BEMERType Pulsed Weak Electromagnetic Field and HPMA-Bound Doxorubicin on Mouse EL4 T-Cell Lymphoma. Journal of Drug Targeting, 19, 890-899. http://dx.doi.org/10.3109/1061186X.2011.622403

[40] Beneduci, A. (2009) Evaluation of the Potential in Vitro Antiproliferative Effects of Millimeter Waves at Some Therapeutic Frequencies on RPMI 7932 Human Skin Malignant Melanoma Cells. Cell Biochemistry and Biophysics, 55, 2532. http://dx.doi.org/10.1007/s12013-009-9053-8

[41] Beneduci, A., Chidichimo, G., Tripepi, S. and Perrotta, E. (2005) Transmission Electron Microscopy Study of the Effects Produced by Wide-Band Low-Power Millimeter Waves on MCF-7 Human Breast Cancer Cells in Culture. Anticancer Research, 25, 1009-1013.

[42] Fedorowski, A., Steciwko, A. and Rabczynski, J. (2004) Low-Frequency Electromagnetic Stimulation May Lead to Regression of Morris Hepatoma in Buffalo Rats. Journal of Alternative and Complementary Medicine, 10, 251-260. http://dx.doi.org/10.1089/107555304323062248

[43] Chidichimo, G., Beneduci, A., Nicoletta, M., Critelli, M., De Rose, R., Tkatchenko, Y., et al. (2002) Selective Inhibition of Tumoral Cells Growth by Low Power Millimeter Waves. Anticancer Research, 22, 1681-1688.

[44] Ufimtsev, I.S., Luehr, N. and Martinez, T.J. (2011) Charge Transfer and Polarization in Solvated Proteins from Ab Initio Molecular Dynamics. Journal of Physical Chemistry Letters, 2, 1789-1793. http://dx.doi.org/10.1021/jz200697c

[45] Boxer, S.G. (1990) Mechanisms of Long-Distance Electron Transfer in Proteins: Lessons from Photosynthetic Reaction Centers. Annual Review of Biophysics and Biophysical Chemistry, 19, 267-299. http://dx.doi.org/10.1146/annurev.bb.19.060190.001411

[46] Kuffel, A. and Zielkiewicz, J. (2013) Properties of Water in the Region between a Tubulin Dimer and a Single Motor Head of Kinesin. Physical Chemistry Chemical Physics, 15, 4527-4537. http://dx.doi.org/10.1039/c3cp43828g

[47] Blank, M. (2008) Protein and DNA Reactions Stimulated by Electromagnetic Fields. Electromagnetic Biology and Medicine, 27, 3-23.

[48] Davis, A., Martinez, S., Nelson, D. and Middleton, K. (2010) A Tubulin Polymerization Microassay Used to Compare Ligand Efficacy. Methods in Cell Biology, 95, 331-351. http://dx.doi.org/10.1016/S0091-679X(10)95018-8

[49] Davis, A., Jiang, J.D., Middleton, K.M., Wang, Y., Weisz, I., Ling, Y.H., et al. (1999) Novel Suicide Ligands of Tubulin Arrest Cancer Cells in S-Phase. Neoplasia, 1, 498-507. http://dx.doi.org/10.1038/sj.neo.7900066

[50] Jiang, J.D., Davis, A.S., Middleton, K., et al. (1998) 3-(Iodoacetamido)-Benzoylurea: A Novel Cancericidal Tubulin Ligand That Inhibits Microtubule Polymerization, Phosphorylates bcl-2, and Induces Apoptosis in Tumor Cells. Cancer Research, 58, 5389-5395. 


\section{Supplement}

Here we describe the technical details of the MIDS system that was used to record magnetic signals from an aqueous paclitaxel solution. We then describe the details of signal processing and the criteria for the selection of the signal M2(3) from among all the signals we acquired and finally we describe the technical details of the transmission of the signal M2(3) to biological samples.

\section{MIDS (Molecular Interrogation and Data System)}

The MIDS system (Figure S1(a) and Figure S1(b)) was designed in collaboration with Tristan Technologies, who constructed and assembled the system (http://tristantech.com/).

Cryogenic part of the MIDS system consists of a 50-liter liquid helium cryogenic Dewar, Model BMD-6 (Tristan Tech, Figure S1(a), cryogen 716). The Dewar is shielded by Mu-metal and additional superconducting electromagnetic shielding (Figure S1(a), MU Shield 718; Figure S1(b), 718, 706) in addition to a lead lining (1a, 1b, 1c).

The Signal acquisition part of the MIDS system is the magnetic sensing package that is surrounded by the Dewar. It consists of a second derivative 4-element gradiometer that is formed from a niobium superconductor and is connected to the input coil of a low temperature (liquid Helium), direct current, superconducting quantum interference device (SQUID, Model LSQ/20 LTS, Figure S1(d), Figure S1(e)). Around the sample well, a two-element Helmholtz transformer is installed orthogonally surrounding the second derivative gradiometer and is connected to a DC offset of $226 \mathrm{mV}$ (Figure S1(b), 726 and 728; Figure S1(e)). The orthogonal placement of the Helmholtz pair renders the input of the DC offset $(226 \mathrm{mV})$ mostly undetectable to the gradiometer. The function of the DC offset is to slightly alter the random orientation of molecules in solution to a more deterministic order. The electric output of the SQUID is connected to a Model SP cryogenic cable (Tristan Tech) that is capable of withstanding the temperatures around the cryogenic Dewar. The cable transfers the signal from the SQUID to a Flux-Locked Loop (model iFL-301-L, Tristan Tech.) mounted externally to the Dewar. The signal then passes over a model CC-60 six meter fiber-optic composite cable (Tristan Tech) to an external iMC-303 iMAG SQUID controller. This fiber-optic cable carries the operator set control signals from the SQUID controller to the Flux-Locked Loop, reducing the possibility of electromagnetic interference of the control currents with the signal to be measured. The SQUID controller undergoes analog to digital conversion via a Keithley KPCI-3108 data acquisition board operating in differential mode. The changes in the magnetic field emitted from the sample are recorded by the SQUID/gradiometer as voltage changes over a 60 second time period and are saved as 24-bit WAV (PCM) files. In 60 seconds, a total of approximately 2.6 million floating points (24 bit) of data are captured. A 20-milli-second window of the water and paclitaxel sample (Figure S3(b), top left and top right, respectively) are illustrative of the data that are recorded. The WAV files are stored digitally and are then used for signal analysis and signal transmission.

\section{Sensitivity of the MIDS System}

The MIDS system allows the detection of very small changes in the magnetic field emitted by molecules suspended in water due to the high level of magnetic "noise" attenuation achieved with the MIDS system (for measuring details see signal analysis below). A power spectrum analysis (Voltage delta Root Mean Square/root Hz, FlexPro8) demonstrates an internal frequency-dependent reduction of the external magnetic background field (Figure S3(a)). A frequency-dependent noise reduction of up to $-100 \mathrm{~dB}$ can be achieved, with an average reduction of $-80 \mathrm{~dB}$ across all frequencies. The second derivative gradiometer and SQUID combination has a theoretical maximum sensitivity of approximately $4 \mathrm{fT}$, with a noise floor of approximately $60 \mathrm{fT}$.

\section{Signal Analysis}

The optimal signal M2(3), containing the highest signal-to-noise ratios and spectral content, was selected from a wide range of measured signals as outlined below. Each recorded 24 bit WAV (PCM) file was analyzed with programs contained in the commercial FlexPro8 package. Mathematical algorithms that were used to identify signal characteristics are described in detail by [1]-[3]. The steps used in signal analysis are outlined in Figure S2 Signal analysis can be divided into the following major steps. 


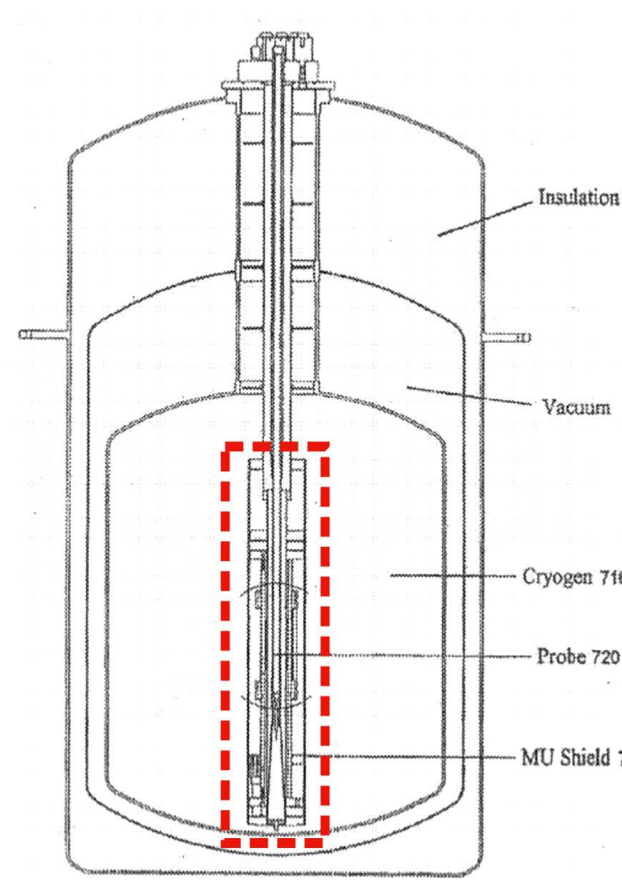

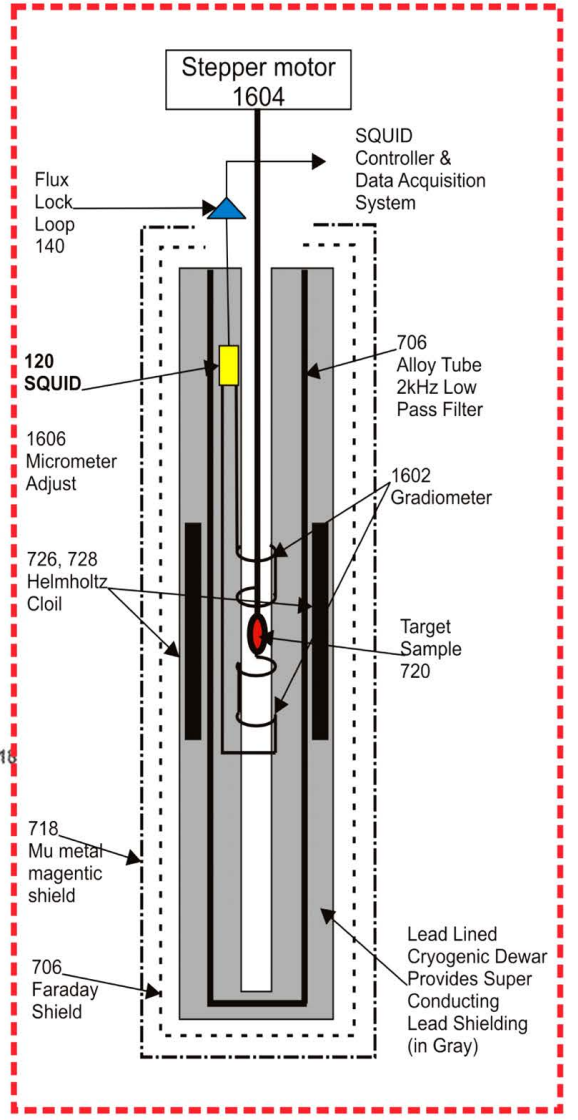

(b)

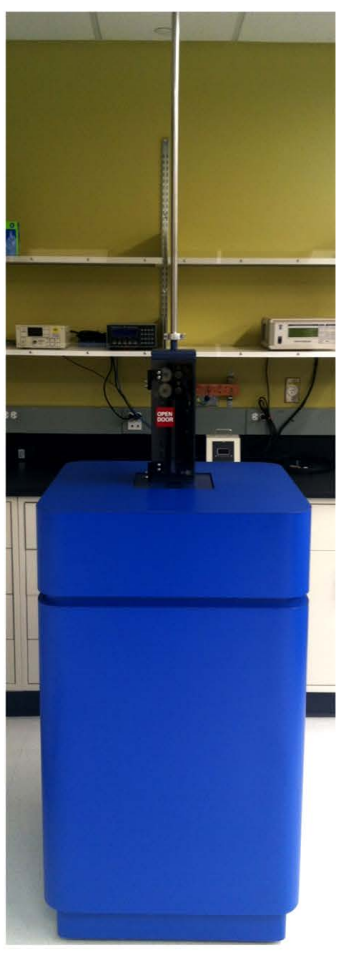

(c)

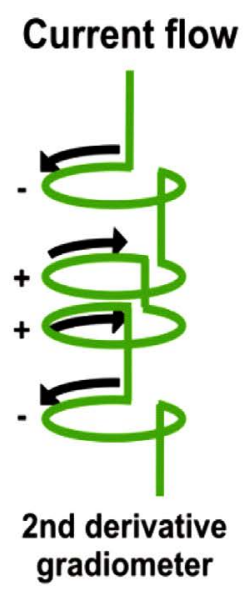

(d)

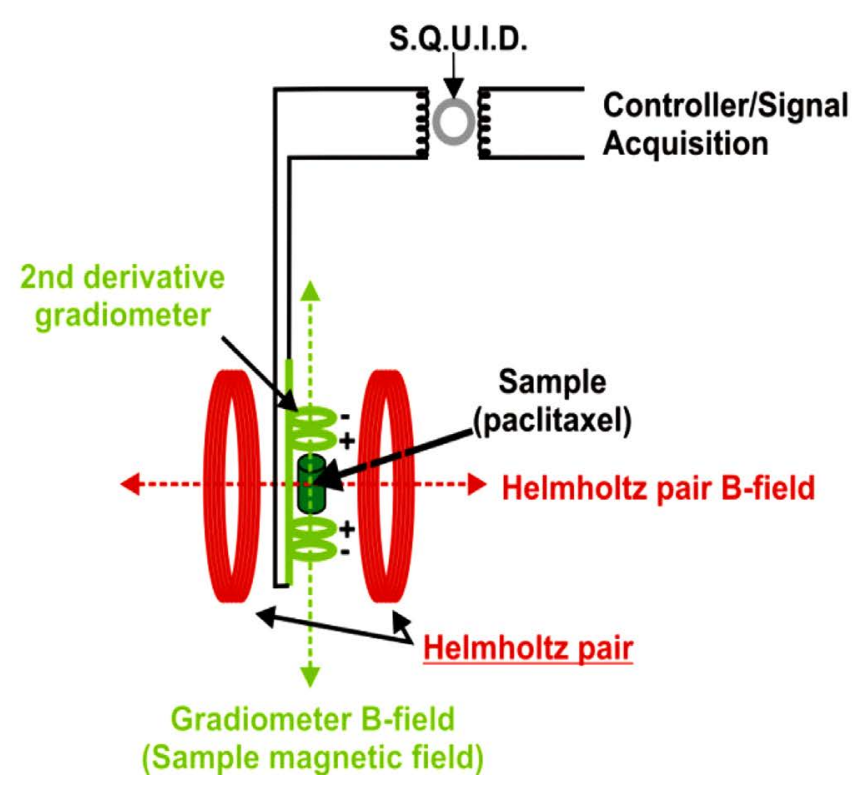

(e)

Figure S1. (a) Construction diagram for the MIDS EMF attenuation chamber and cryogenic Dewar; (b) Diagram of the SQUID/gradiometer sample analyzer set-up inside the cryogenic Dewar; (c) Assembled system; (d) Representative diagram and current flow (black curved arrows) for noise cancellation in the second derivative gradiometer; (e) Representative diagram of Helmholtz pair in relationship to the second derivative gradiometer and the direction of the B-fields for the Helmholtz pair (red line with arrows) and second derivative gradiometer detection field (green line with arrows). 


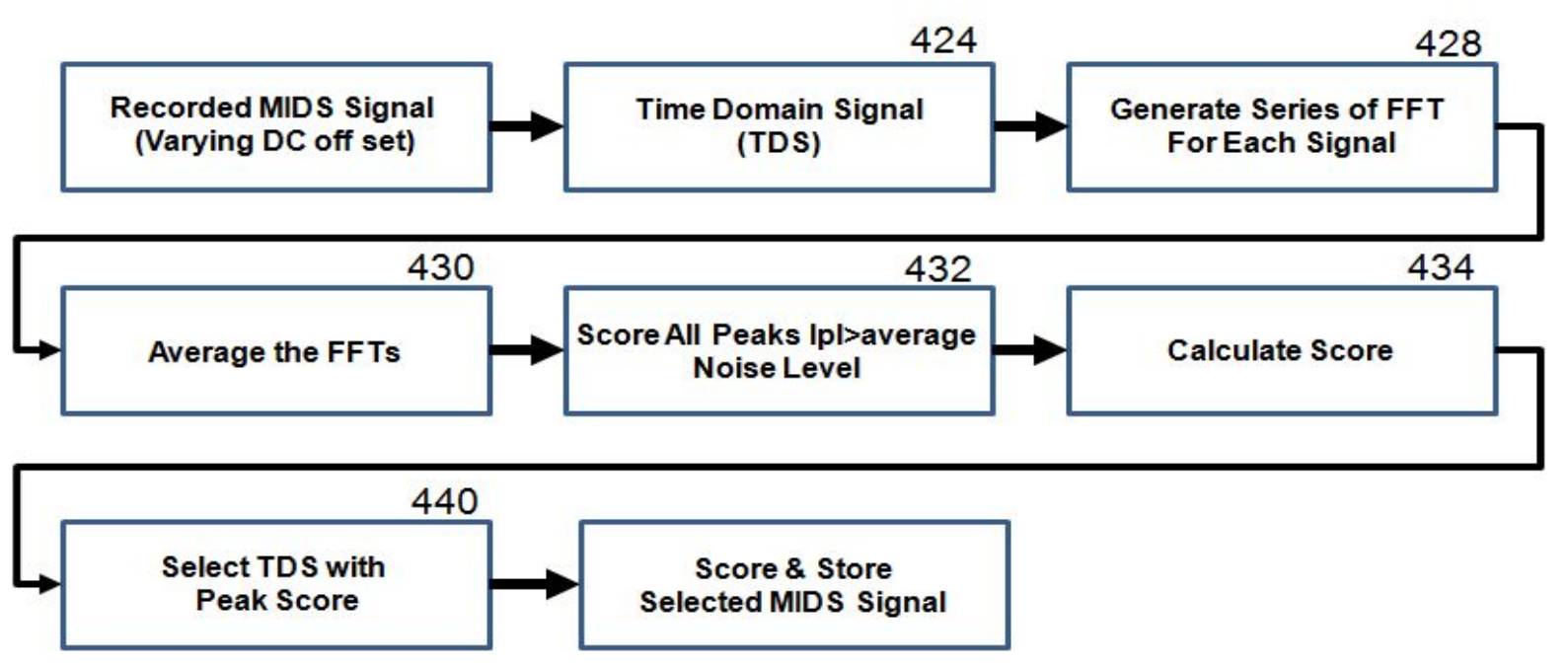

Figure S2. Signal Analysis: Flow diagram of analysis process in signal selection after MIDS data acquisition from a sample.

\subsection{Autocorrelation}

Flexpro8 first performs a normalized autocorrelation within the time domain for each signal (Figure S2, Box 424). This generates a discrete sampled, auto-correlated time-domain signal from the initial WAV file. The auto-correlated time series is 60 seconds long (Sample Duration), with a rate of 44,100 samples per second, thus providing a total of 2,646,000 (Sample Duration * Sample Rate) samples. The Frequency Range that can be recovered from the signal is half the Sample Rate (Nyquist). Hence, with 44,100 samples per second, the Frequency Range we cover is $0 \mathrm{~Hz}$ to $22 \mathrm{kHz}$.

\subsection{Fast Fourier Transform (FFT)}

An FFT, using a Radix 2 Real Fast Fourier Transform (RFFT) (Flexpro8), was used to convert the signal to a frequency domain spectrum for further analysis (Figure S2, Box 428). An FFT Size of 8192 was selected to provide enough resolution for at least one spectrum bin per Hertz for the Frequency Range at or below $8 \mathrm{kHz}$. The Sample Duration of 60 seconds was long enough (Sample Count > (2) * FFT Size * 10) to ensure reliable results. We chose to overlap the FFT input by half the FFT Size, which reduces the error to $1 /(0.81 * 2 *$ FFT Size) $\wedge$ 0.5. This reduces the statistical error from 0.0110485435 to 0.0086805556 (For additional information about errors and correlation analyses in general, consult [4]). Note that (FFT Size) = 8192 is the resolution, or is the number of bins in the range dictated by the sampling rate. The variable $\mathrm{n}$, which dictates how many discrete RFFTs (Real FFTs) performed, is set by dividing the Sample Count by (FFT Size) * 2, the number of FFT bins. We have chosen 8192 for the variable (FFT Size), which allows us to pick up weaker signals (based on bin resolution, $\mathrm{F}_{S} / \mathrm{N}$, where $\mathrm{F}_{\mathrm{S}}$ is the input signal's sampling rate and $\mathrm{N}$ is the number of FFT points used.) The program initializes the histograms and arrays and copies (FFT Size) samples of the wave data into buffers, and performs an RFFT on the wave data. The FFT is normalized so that the highest amplitude is 1 and the average power for all bins (FFT Size) is determined from the normalized signal. For each bin frequency, the normalized value from the FFT at that frequency is added to each bin in its buffer.

The program RFFT then looks at the power at each bin frequency, relative to the average power calculated from nearby bins. If the power is within a certain factor epsilon (between 0 and 1) of the average power, then it is counted and the corresponding bin is incremented in the histogram; otherwise, if the power is outside of 0 or 1 , the corresponding bin is discarded.

In summary, the Radix 2 Real Fast Fourier Transform program performs the FFT on the samples sequentially and averages the results together for the final spectrum (Figure S2, Box 430; see Figure S3(c) and Figure S4, for a typical output at this stage.)

\subsection{Histogram Method of Generating Spectral Information}

After RFFT, the different signals were compared to each other in a line plot (Figure S2, Box 432) and/or after 


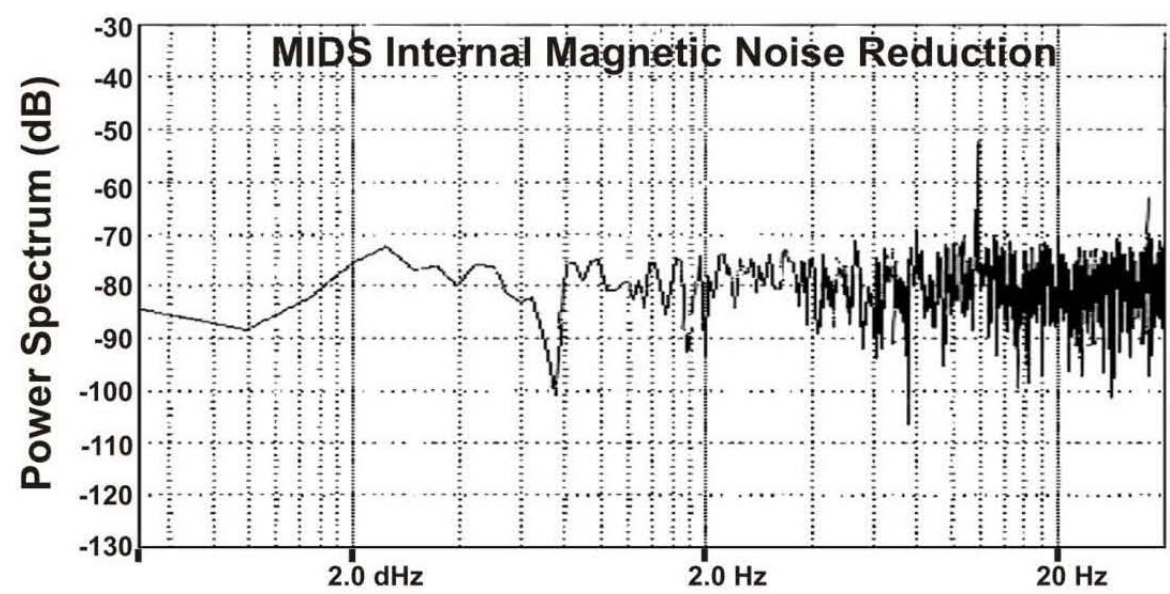

(a)

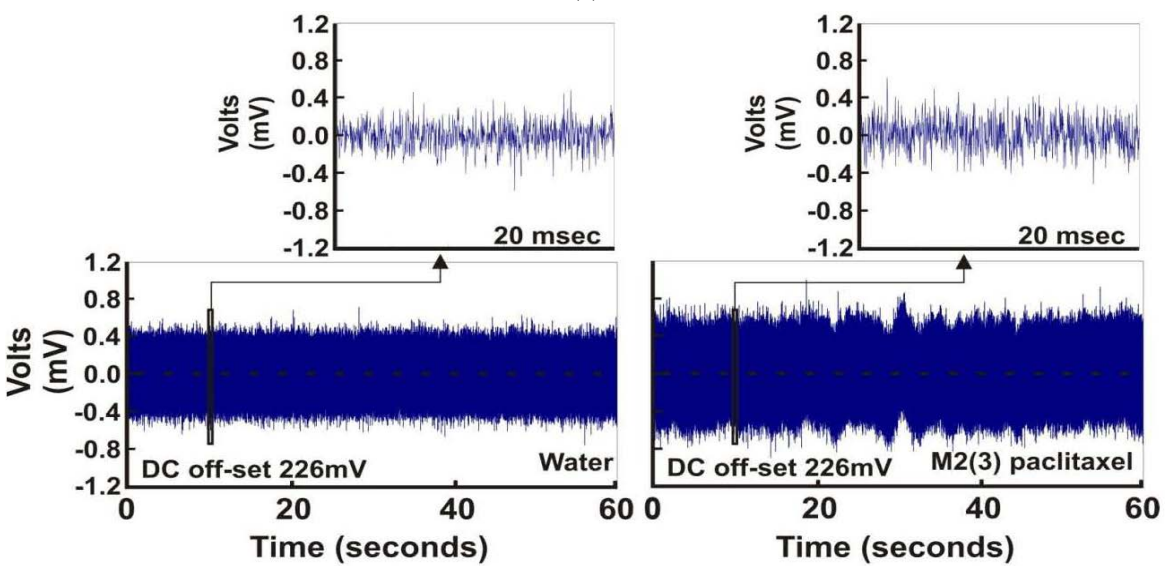

(b)

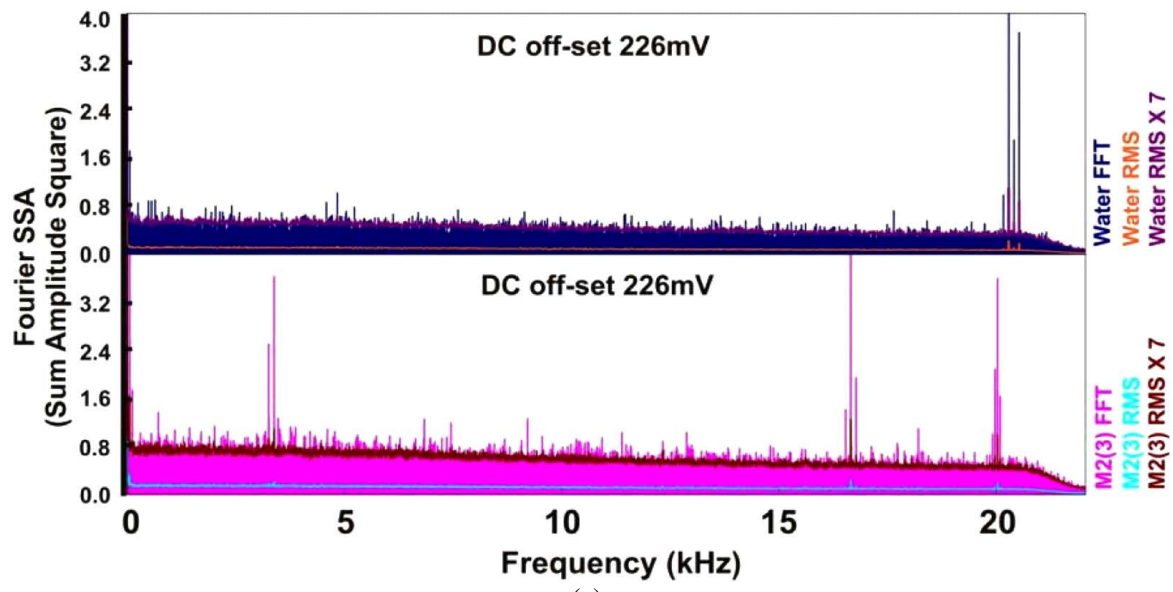

(c)

Figure S3. Measurement system for MIDS. (a) Power spectrum (dB) to frequency of AC magnetic field reduction inside the MIDS. A plot of $\mathrm{dB}$ reduction (Voltage root means square/root $\mathrm{Hz}$ ) shows the relationship between frequency and the attenuation of measured magnetic field strength; (b) Recorded signal from MIDS of water (top) and paclitaxel in water (bottom). The paclitaxel signal was used in our experiment described in the manuscript. The sixty second recordings were selected from 18 hours of recording from the MIDS. Approximately 2.6 million ( 24 bit) floating points were captured in the 60 second recoding for each sample; (c) Comparison of Fourier Sum Amplitude Square versus frequency in samples of water and paclitaxel. Root mean square (RMS), fast Fourier transform (FFT) and RMS $\times$ 7 were used to identify signals in our samples. 


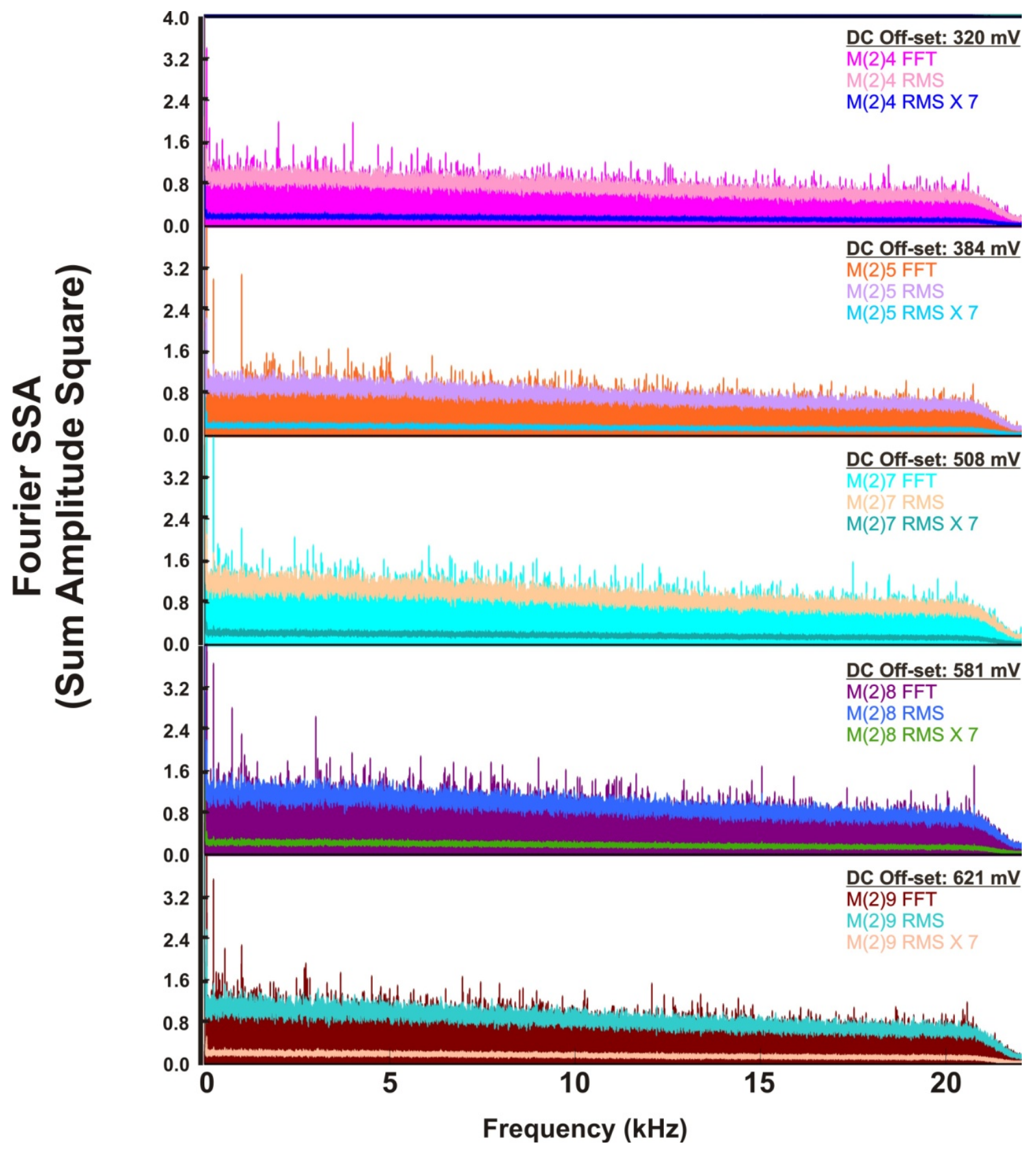

Figure S4. Fourier analysis of non-selected paclitaxel signals. Five different, 60 second signal recordings of a single paclitaxel sample with different DC off-set applied. The individual signals display variations in the Fourier analysis. Signals were determined to be inferior due to low power to entropy ratio across the spectrum and initial testing of samples.

applying the root mean square (RMS) analysis on the FFT output (see Figure S3(c); upper graph, water; lower graph, M2(3) paclitaxel signal). Other paclitaxel signals that were not chosen (Figure S4) are presented for comparison. A Fourier SSA, sum amplitude square analysis was performed on various signals presented in the supplement to show signal content and to differentiate between different signals. The bin histogram produces two types of data, one representing information in the frequency domain, and a score value representing the overall power contained in all bins over epsilon. The power over epsilon was used to help identify candidate signals. 
The histogram (Figure S3(c) and Figure S4) produced by the RFFT contains in each bin the number of times that the power at that frequency was above [epsilon * the average power for that whole FFT output; epsilon = 1.2]. If a bin count is incremented due to unstructured noise, that noise will be distributed across all the frequency bins over time, thus not amounting to much in a given bin. If there is consistent signal at a given frequency, it will be present at each of the $\mathrm{n}$ time slices and thus have a bin count approaching $\mathrm{n}$. Large amplitude noise, such as sixty Hertz and its harmonics have both high bin counts as well as high average power. We can differentiate between the inherent noise frequencies and the ones in which we are interested, which have low average power but high bin counts (compare Figure S3(c), upper panel with lower panel and lower panel of Figure S3(c) with Figure S4).

\section{Criteria for Selection of M2(3) from All Other Signals}

M2(3) was selected because it had a high histogram index (score), high signal to noise, low entropy and low artifact. (Figure S3(c), lower panel, compared to the rejected signals (Figure S4). The original WAV file of M2(3) recorded from the MIDS was then used for signal transmission to the MIDS. No alterations were made to the WAV file.

\section{Signal Transmission to Biological Samples}

Voyager VX (experimental prototype) was designed and constructed in house. Voyager VX (Figure S5(a), Figure S5(b)) converts the digital time domain signal (WAV file recorded from the MIDS) into current pulses. These current pulses were transmitted to the Helmholtz coils that surround the tubulin assay mixture. Voyager VX consists of a pre-amplifier (Black box on top; Creative Labs, model No. SB1090)/amplifier; Figure S5(a), 556; Figure S5(b)) connected to a control unit (552) that puts out an amplified signal voltage to an attenuator (558), also under the control of unit 550. The attenuator converts the signal voltage generated by the amplifier (556) to a current that is supplied to the transducer Helmholtz coils to produce a selected range of magnetic fields (see Coil design below). Output functions and field strengths are set at the Voyager VX and are displayed on 553 (Figure S5(b), zoomed portion). The Voyager VX was set to achieve incremental uniform gradient magnetic fields from about $0.1 \mathrm{nT}$ to $10 \mu \mathrm{T}$, over 42 increments, each setting was maintained for 1 second and the system continuously cycled through the range of field strengths over a $10 \mathrm{~min}$ period. The signal was supplied to the Helmholtz coils (Specifications see below) (562 and 564, Figure S5(a)). The Helmholtz coils are housed inside a Mu-metal chamber (Figure S6(a) and Figure S6(b)). The experimental sample was monitored by a photo spectrometer over an optical cable (568 and 570) connected to a spectrometer to measure the effects of the signal.

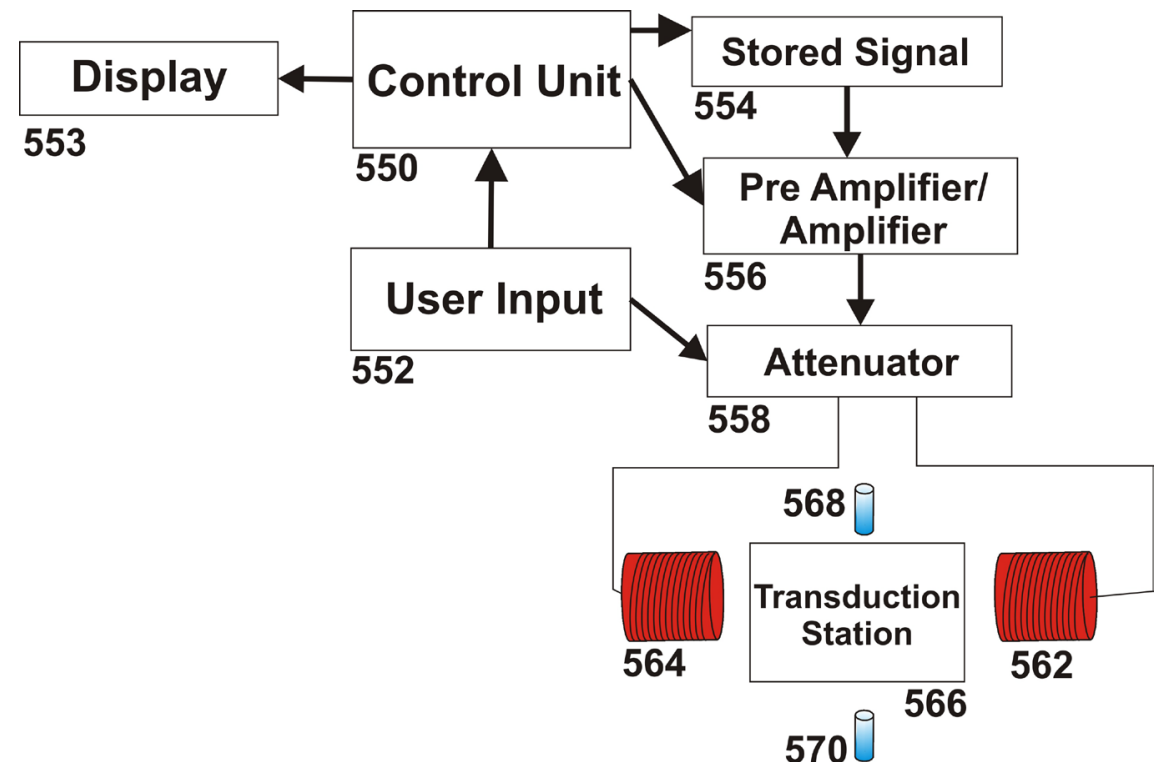

(a) 


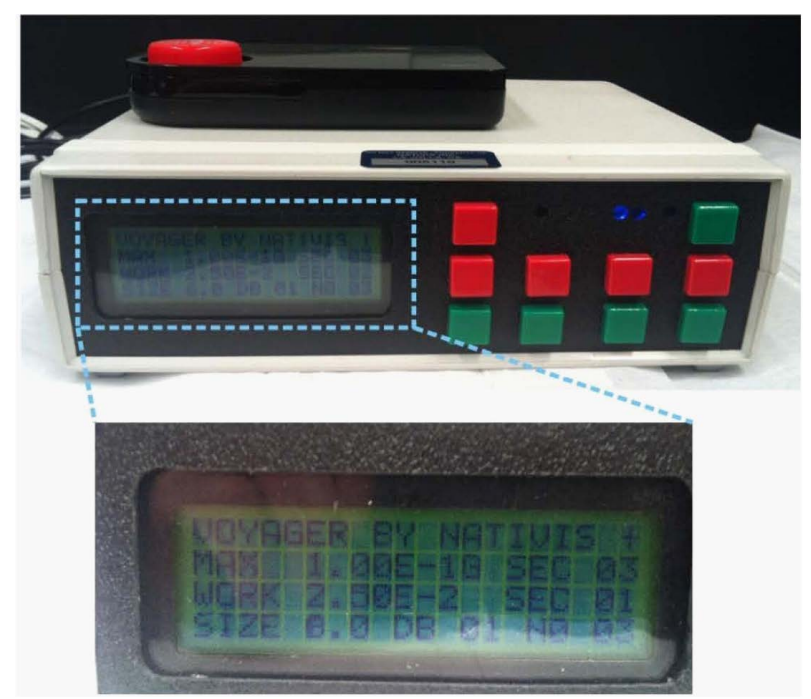

(b)

Figure S5. Signal delivery for taxane signal. (a) Diagrammatic representation of signal delivery set up and components. Numbers 568 and 570 represent the optical path for the spectrophotometer. Numbers 553 - 558 are integral components of the Voyager VX unit; (b) Prototype system with integral electronics and amplifier: Voyager VX (inset, LCD unit display of power settings).
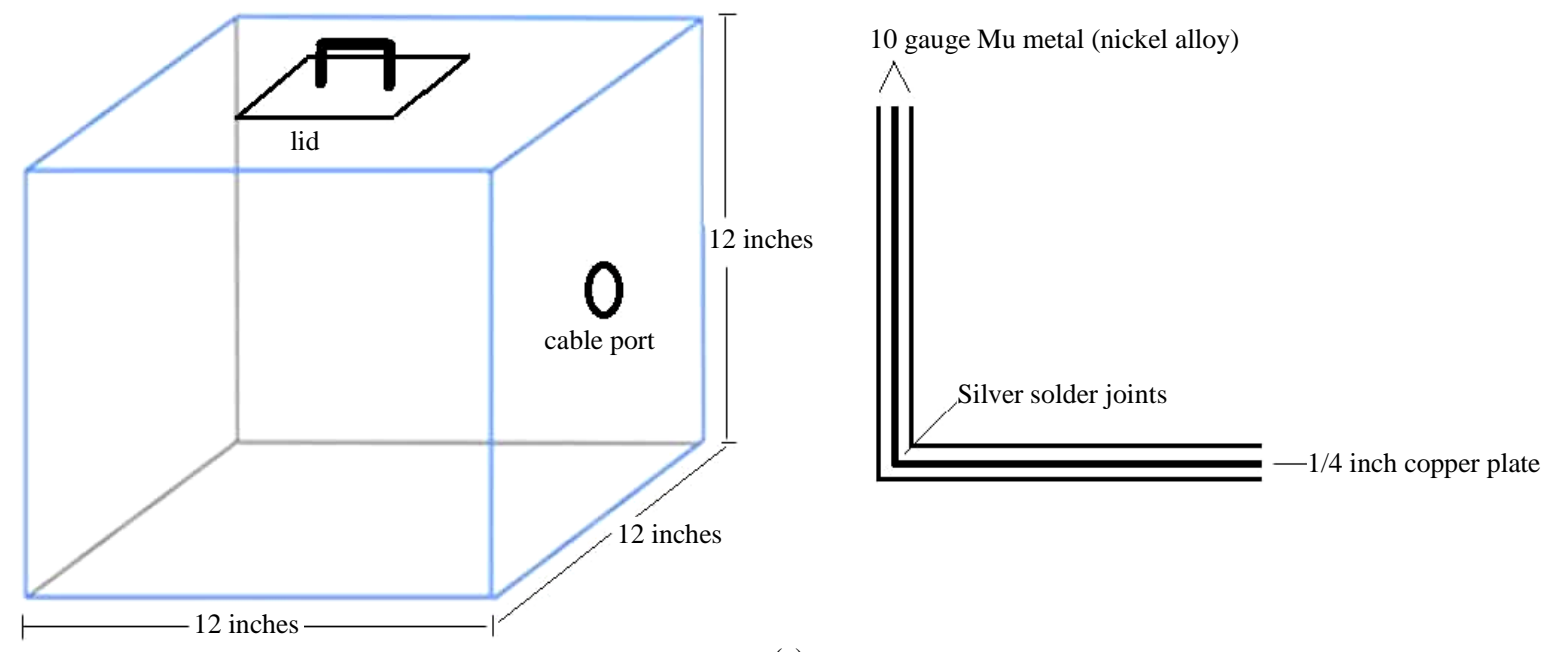

(a)

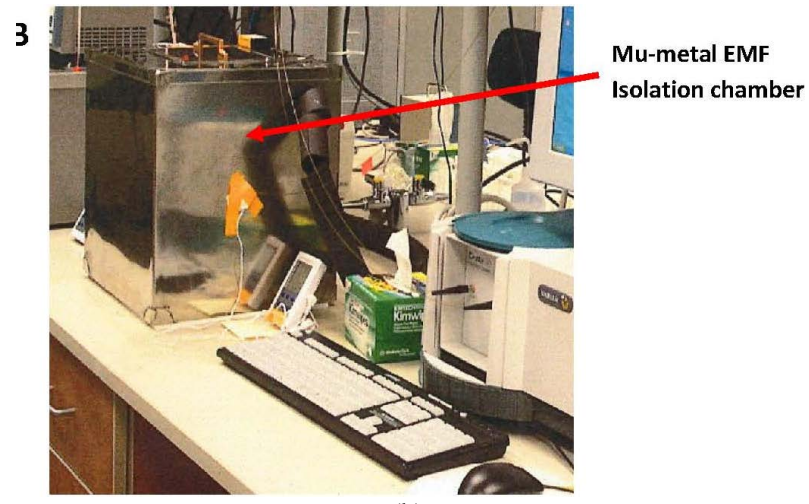

(b)

Figure S6. Mu-metal Isolation Chamber. Schematic diagram of EMF isolation chamber (a) and (b) EMF isolation chamber set-up for visual spectrometer measurements. 

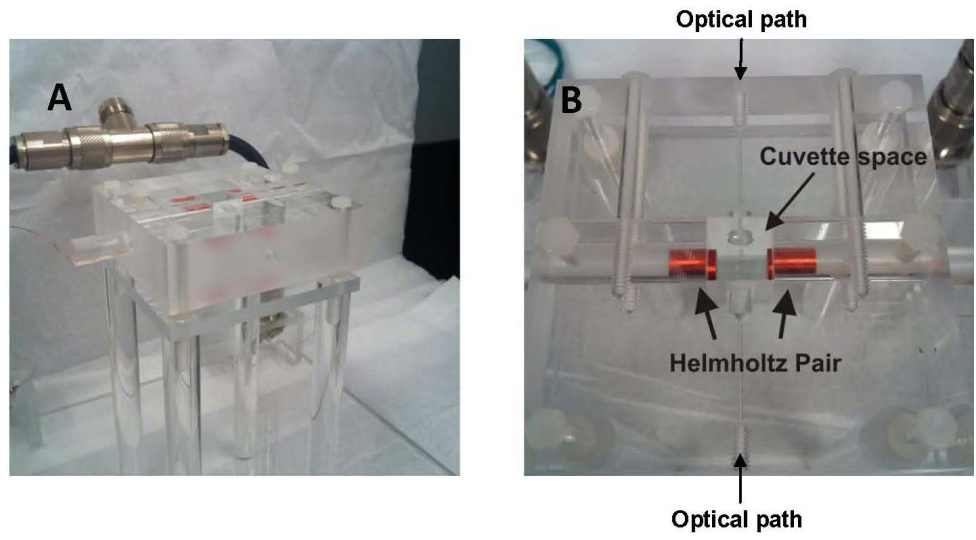

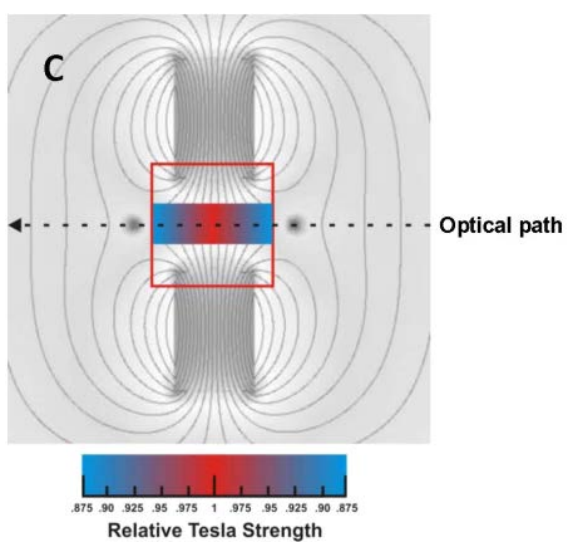

Figure S7. Helmholtz's pair platform for spectrometer measurements: (A) Acrylic cuvette holder with integrated Helmholtz coil pairs; (B) Overhead view of Helmholtz coils within the cuvette holder. The cuvette holder was made in house and placed inside the Mu-metal chamber shown in Figure S6. Optical path in cuvette holder is diagrammed in Figure S6(a), \#568 and \#570; (C) The modeled magnetic field generated by the Helmholtz pair. The red box between the Helmholtz pair represents the external dimensions of the cuvette. The internal, color gradient rectangle represents the cuvette volume that contains the sample for exposure and measurement. The color gradient scale (below the field model) represents the relative Tesla strength (intensity) of the applied field to the sample. The dashed black arrow represents the light path of the OD measurement.

\section{Coil Design and Coil Shielding}

The signal was transmitted to a pair of $8 \mathrm{Ohm}$ Helmholtz coils. The Helmholtz pairs were 8.5 millimeters in diameter, positioned at opposite sides of the cuvette with the tubulin assay sample (Figure S7(b)). The distance from coil edge to coil edge relative to the samples was 1 radius. The holder was placed inside the EMF isolation chamber (Figure S6(b)). All measurements were performed in this set-up. The electromagnetic field that is generated in the Helmholtz coils can be modeled (Figure S7(c)) and has well characterized field strength. The image is a stock reference to the same Helmholtz geometry we use. The engineering of our coil pair was based on the standard Helmholtz equation with a separation between coils of one radius. The accuracy $(+/-1 \%)$ for the strength of the of the field (nT) is described for the center one-third of the diameter between the coils. The $70 \mu \mathrm{l}$ cuvette has a narrow channel in the center one-third in order to maximize the light path length for measurement for optical density. Disposable spectrometer cuvettes were placed within the custom built holder (Figure S7(a)) with the tubulin polymerization assay samples in the center of the Helmholtz coil where they were exposed for 10 minutes to the signal (Figure S7(b)).

\section{Supplement References}

[1] Zwillinger, D. (2012) Standard Mathematical Tables and Formulae. 32nd Edition, CRC Press, Taylor \& Francis Group, Boca Raton.

[2] Weisstein, E.W. Cross-Correlation. From MathWorld-A Wolfram Web Resource. http://mathworld.wolfram.com/Cross-Correlation.html

[3] Grafakos, L. (2004) Classical and Modern Fourier Analysis. Prentice-Hall, Upper Saddle River.

[4] Bendat, J.S. and Piersol, A.G. (1993) Engineering Applications of Correlation and Spectral Analysis. Wiley, New York. 
Scientific Research Publishing (SCIRP) is one of the largest Open Access journal publishers. It is currently publishing more than 200 open access, online, peer-reviewed journals covering a wide range of academic disciplines. SCIRP serves the worldwide academic communities and contributes to the progress and application of science with its publication.

Other selected journals from SCIRP are listed as below. Submit your manuscript to us via either submit@scirp.org or Online Submission Portal.
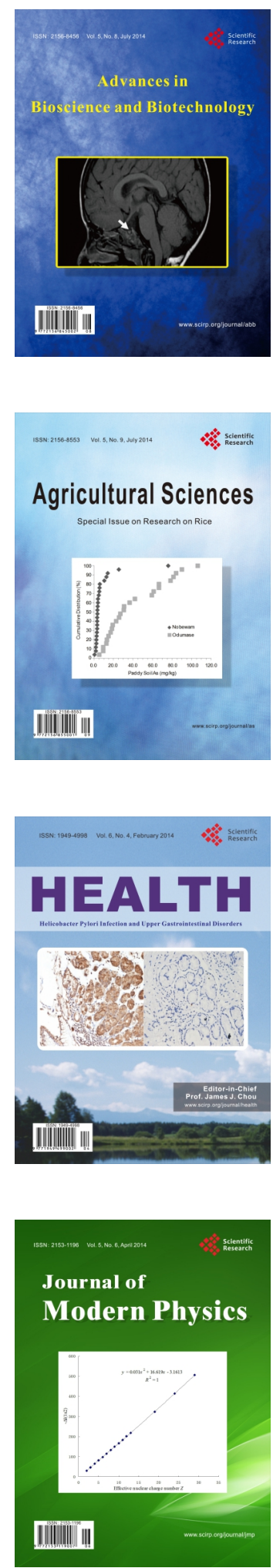
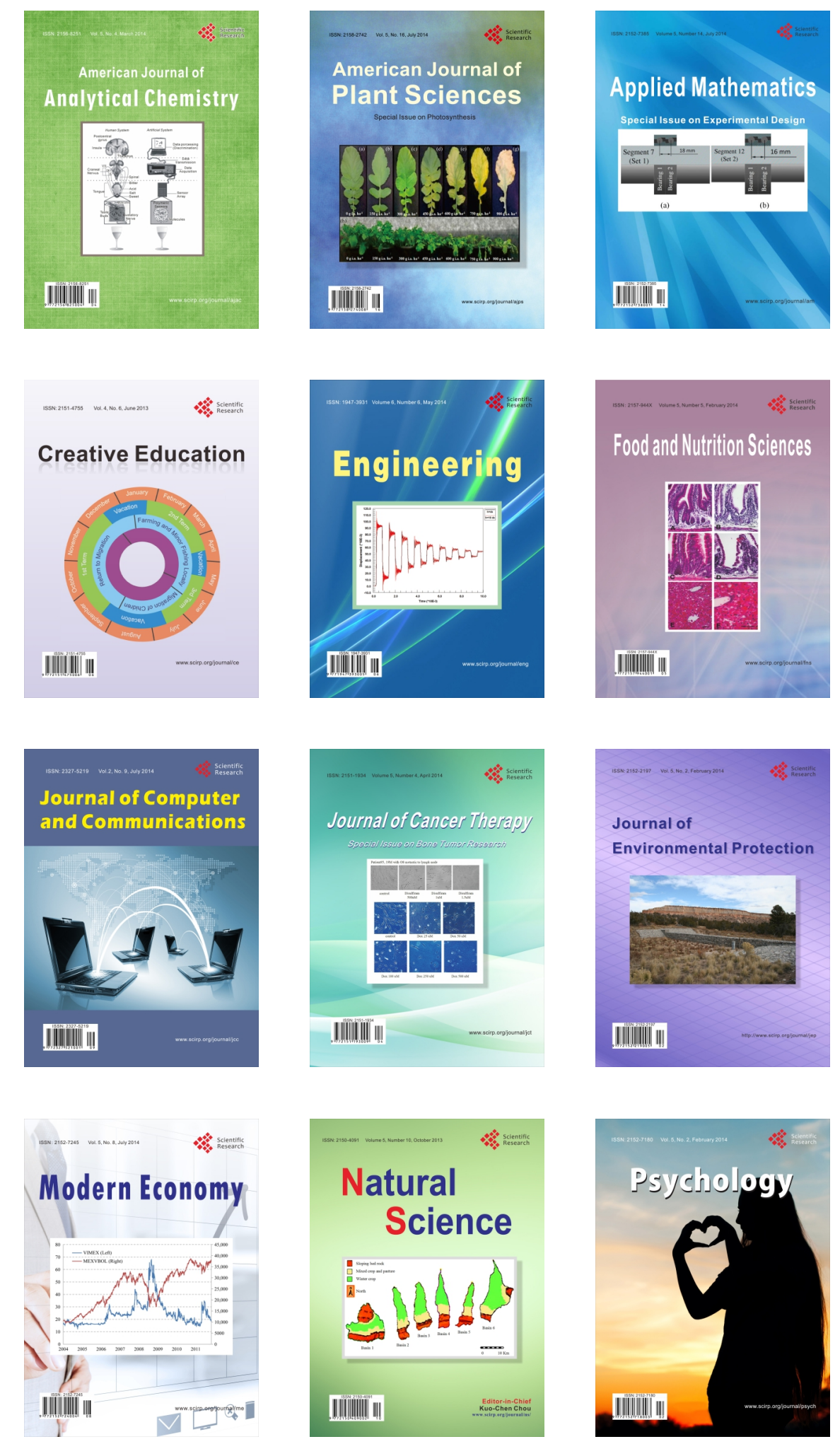